\title{
Scaling Regimes of Active Turbulence with External Dissipation
}

\author{
Berta Martínez-Prat $\odot,{ }^{1,2, *}$ Ricard Alert $\odot,{ }^{3,4, *}$ Fanlong Meng $\odot,{ }^{5,6,7}$ Jordi Ignés-Mullol@ ${ }^{1,2}$ Jean-François Joanny, ${ }^{8,9,10}$ \\ Jaume Casademunt, ${ }^{11,12}$ Ramin Golestanian $\oplus^{5,13}$ and Francesc Sagués $\oplus^{1,2}$ \\ ${ }^{1}$ Department of Materials Science and Physical Chemistry, Universitat de Barcelona, \\ Barcelona, Catalonia 08028, Spain \\ ${ }^{2}$ Institute of Nanoscience and Nanotechnology (IN2UB), Universitat de Barcelona, \\ Barcelona, Catalonia 08028, Spain \\ ${ }^{3}$ Princeton Center for Theoretical Science, Princeton University, Princeton, New Jersey 08544, USA \\ ${ }^{4}$ Lewis-Sigler Institute for Integrative Genomics, Princeton University, Princeton, New Jersey 08544, USA \\ ${ }^{5}$ Max Planck Institute for Dynamics and Self-Organization (MPIDS), D-37077 Göttingen, Germany \\ ${ }^{6}$ CAS Key Laboratory for Theoretical Physics, Institute of Theoretical Physics, \\ Chinese Academy of Sciences, Beijing 100190, China \\ ${ }^{7}$ School of Physical Sciences, University of Chinese Academy of Sciences, Beijing 100049, China \\ ${ }^{8}$ ESPCI Paris, PSL University, Paris, France \\ ${ }^{9}$ Laboratoire PhysicoChimie Curie, Institut Curie, PSL University, \\ Sorbonne Universités, UPMC, Paris, France \\ ${ }^{10}$ Collège de France, Paris, France \\ ${ }^{11}$ Departament de Física de la Matèria Condensada, Universitat de Barcelona, \\ Barcelona, Catalonia 08028, Spain \\ ${ }^{12}$ Universitat de Barcelona Institute of Complex Systems (UBICS), Universitat de Barcelona, \\ Barcelona, Catalonia 08028, Spain \\ ${ }^{13}$ Rudolf Peierls Centre for Theoretical Physics, University of Oxford, Oxford OX1 3PU, United Kingdom
}

(Received 22 March 2021; revised 24 June 2021; accepted 30 July 2021; published 23 September 2021; corrected 20 October 2021 and 17 May 2022)

Active fluids exhibit complex turbulentlike flows at low Reynolds number. Recent work predicted that 2D active nematic turbulence follows scaling laws with universal exponents. However, experimentally testing these predictions is conditioned by the coupling to the $3 \mathrm{D}$ environment. Here, we measure the spectrum of the kinetic energy $E(q)$ in an active nematic film in contact with a passive oil layer. At small and intermediate scales, we find the scaling regimes $E(q) \sim q^{-4}$ and $E(q) \sim q^{-1}$, respectively, in agreement with the theoretical prediction for 2D active nematics. At large scales, however, we find a new scaling $E(q) \sim q$, which emerges when the dissipation is dominated by the 3D oil layer. In addition, we derive an explicit expression for the spectrum that spans all length scales, thus explaining and connecting the different scaling regimes. This allows us to fit the data and extract the length scale that controls the crossover to the new large-scale regime, which we tune by varying the oil viscosity. Overall, our work experimentally demonstrates the emergence of scaling laws with universal exponents in active turbulence, and it establishes how the spectrum is affected by external dissipation.

DOI: $10.1103 /$ PhysRevX.11.031065

\section{INTRODUCTION}

Active fluids are able to flow spontaneously due to the internal stresses that are generated by their microscopic

\footnotetext{
"These authors contributed equally to this work.

Published by the American Physical Society under the terms of the Creative Commons Attribution 4.0 International license. Further distribution of this work must maintain attribution to the author(s) and the published article's title, journal citation, and DOI. Open access publication funded by the Max Planck Society.
}

Subject Areas: Nonlinear Dynamics, Soft Matter, Statistical Physics 
emergent universal behavior and scaling laws $[34,35]$ in active turbulence.

These issues have been the subject of intense research and debate $[5,8,12,13,33,36-45]$. Inspired by bacterial turbulence, initial work on extended Toner-Tu equations for polar flocks showed that these fluids exhibit flow spectra with scaling regimes. However, the corresponding scaling laws have nonuniversal exponents, whose values depend on model parameters [12,38,39,46-49]. In contrast, recent theoretical work on active nematics predicted scaling laws with universal exponents, which are independent of the active fluid properties (e.g., viscosity or activity) [36,37]. Experimental evidence for such universal scaling, however, remained elusive, in part due to the challenge of accurately measuring flows for sufficiently broad ranges of scales.

In addition, most of the theoretical predictions and numerical studies have addressed the case of two-dimensional flows. As in classical turbulence, 2D systems are often embedded in a 3D setup. It is, therefore, necessary to separate the genuinely $2 \mathrm{D}$ features from the effects due to the coupling to the environment. In classical 2D turbulence, for instance, frictional dissipation with the environment not only cuts off the energy cascade toward large scales, but it also modifies the scaling exponent of the enstrophy cascade toward small scales [50,51].

Here, we address the influence of external dissipation on the scaling regimes in microtubule-based active nematics powered by motor proteins $[19,24]$. In this setup, the 2D active nematic is surrounded by layers of oil and water, two passive fluids. Our comprehensive experimental study spans a broad range of spatial scales and includes systematic variation of the oil viscosity. We also present a theoretical framework that incorporates the hydrodynamic coupling with the environment and provides an explicit expression for the full spectrum of the turbulent active flows. Combining theory and experiments, our results provide evidence of scaling laws with universal exponents in $2 \mathrm{D}$ active turbulence and determine the ranges where these scaling laws are observable in terms of the physical parameters.

Our predictions include six different scaling regimes, which we classify in terms of three length scales: the average vortex size, the height of an external fluid layer, and a viscous length that controls whether dissipation is dominated by either the active or the external fluid. This analysis reveals that, in contrast to classic turbulence, external dissipation does not just introduce a small- $q$ cutoff to the scaling behavior, but it also yields a new scaling regime. In our experiments, we vary the oil viscosity over more than 4 orders of magnitude and observe three of the predicted scaling regimes. The remaining regimes might be observed either in alternative experimental setups or in other systems such as cell monolayers.

Beyond the scaling laws, the detailed comparison between theory and experimental data allows us to probe other open questions in active turbulence. Specifically, we seek to explain the emergence of a characteristic vortex size. The coupling to external fluids selects a characteristic wavelength at the onset of spontaneous flows [24,53-55]. However, we show that this selection mechanism cannot fully account for the average vortex size observed in the turbulent regime. Thus, our analysis suggests that nonlinear effects in the active fluid, possibly involving energy transfer across scales, also contribute to vortex size selection. Moreover, we establish the range of validity of our theory, as we observe that it correctly fits the data for an intermediate range of oil viscosities but fails for extremely low and high viscosities. Our measurements suggest directions for future improvements to the theory, such as including vortex-vortex correlations.

\section{EXPERIMENTAL SETUP AND ENERGY SPECTRA}

In our experiments, we prepare an active nematic film by self-assembly of micrometer-long stabilized microtubules at the interface between a $H_{\text {water }} \approx 40-\mu$ m-thick water layer on a glass slide and a $H_{\text {oil }} \approx 3$-mm-thick oil layer open to the air [56] [bottom inset in Fig. 1(a) and Appendix A]. The microtubules are bundled under the depleting action of polyethylene-glycol (PEG), which facilitates cross-linking by kinesin molecular motors clustered with streptavidin (Appendix A). Fueled by adenosine triphosphate (ATP), the motors generate active shear stresses leading to extension and buckling of the microtubule bundles. The bundles then acquire local nematic order interrupted by half-integer topological defects, and the film exhibits disordered large-scale flows, which is the state of active nematic turbulence (see Movie 1 in Supplemental Material [52]).

To study the statistical properties of active turbulent flows, we measure their so-called kinetic energy spectrum $E(q) \propto q\left\langle|\tilde{\boldsymbol{v}}(q)|^{2}\right\rangle$, where $\tilde{\boldsymbol{v}}(\boldsymbol{q})$ are the Fourier components of the flow field $\boldsymbol{v}(\boldsymbol{r})$, with wave vector $\boldsymbol{q}$ (Appendix A). To explore the different flow regimes, we vary an external parameter, namely, the oil viscosity, while keeping the intrinsic active fluid parameters-including motor, microtubule, and ATP concentrations-fixed. Therefore, the socalled active length, which compares the active and elastic stresses, is kept constant in all of our experiments. Upon increase of the oil viscosity, the entire kinetic energy spectrum decreases (Fig. 1), which is consistent with the previously observed decrease of flow speed [56]. At low oil viscosities, the spectrum features at least three regimes: a large-scale (small- $q$ ) regime that is followed by a peak, an intermediate regime, and a crossover to a small-scale (large- $q$ ) regime [Fig. 1(a)]. As oil viscosity increases, the peak shifts to smaller scales, expanding the range of the large-scale regime and shrinking the intermediate regime until it can no longer be observed for high oil viscosities [Figs. 1(b) and 1(c)]. In parallel to these changes in the flow properties, we also observe a higher density of defects for higher oil viscosities (top insets in Fig. 1). 
(a)

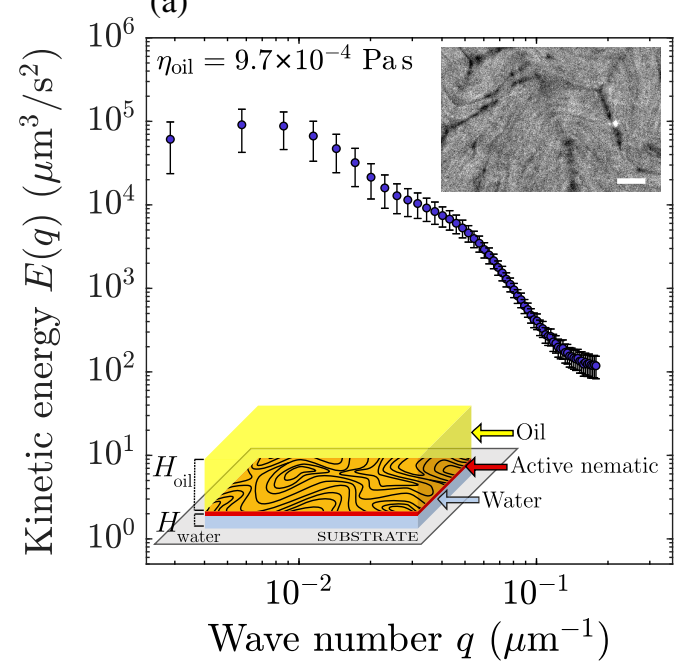

(b)

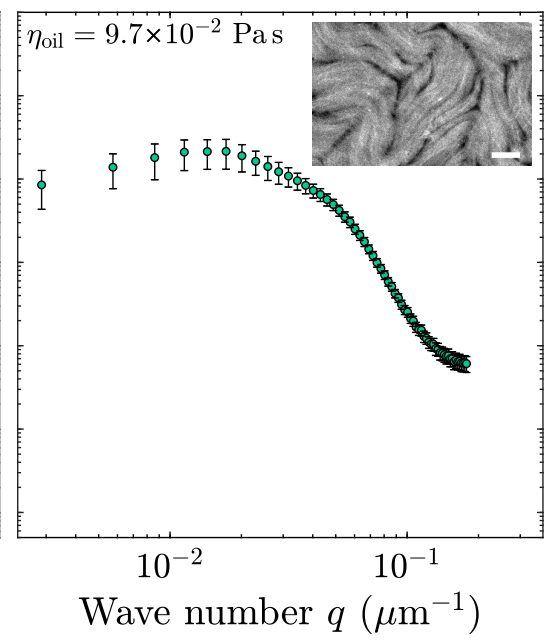

(c)

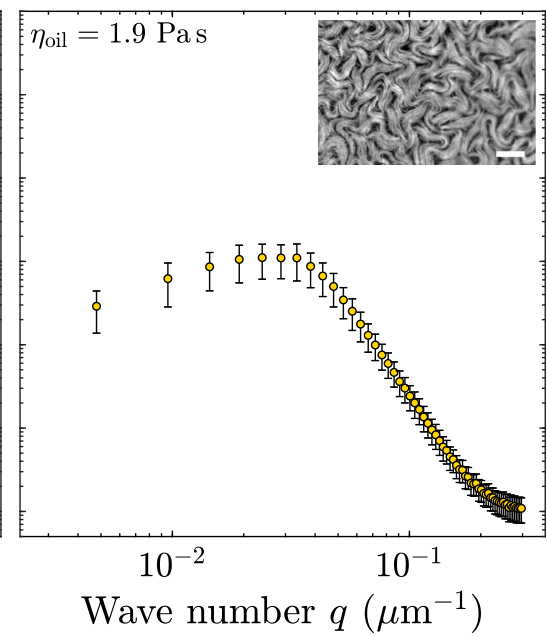

FIG. 1. Oil viscosity modifies the kinetic energy spectrum of active nematic turbulence. (a)-(c) Kinetic energy spectra of turbulent flows in an active nematic film in contact with an oil layer of low (a), intermediate (b), and high (c) viscosity. Averages are over 500 frames. Error bars are standard deviations. In each panel, the top inset shows a representative microtubule fluorescence micrograph. Scale bar is $100 \mu \mathrm{m}$. The bottom inset in (a) shows a schematic of the experimental system. See also Movie 2 in Supplemental Material [52].

\section{THEORETICAL MODEL AND PREDICTED SCALING REGIMES}

To understand our measured spectra, we develop a theoretical framework that accounts for the hydrodynamic coupling between the active film and the external water and oil layers, with their corresponding boundary conditions. Active flows in the nematic film induce flows in the passive layers which, in turn, influence the active-film flows (Fig. 5 in Appendix B). Generalizing previous works [56-60], we first obtain the Green function of the active flows subject to this feedback [Appendix B 1; see Eq. (B11)]. We then establish a relationship between the velocity power spectrum in our coupled three-fluid system and the vorticity power spectrum of an isolated active nematic film [Appendix B 2; see Eq. (B22)]. The latter was previously predicted by Giomi using a mean-field theory approach based on decomposing the vorticity field into a superposition of $N$ uncorrelated vortices [36]. Based on simulation results, Giomi's theory assumes that each vortex has a uniform and size-independent vorticity $\omega_{v}$, and that vortex areas follow an exponential distribution with mean $a_{*}=\pi R_{*}^{2}$, where $R_{*}$ is the mean vortex radius. For a configuration that has on average $N$ vortices over a total system area $\mathcal{A}$, we predict a kinetic energy spectrum (Appendix B 2)

$$
E(q)=\frac{B q R_{*}^{4} e^{-q^{2} R_{*}^{2} / 2}\left[I_{0}\left(q^{2} R_{*}^{2} / 2\right)-I_{1}\left(q^{2} R_{*}^{2} / 2\right)\right]}{\left[q+\eta_{\mathrm{oil}} / \eta_{n} \tanh \left(q H_{\mathrm{oil}}\right)+\eta_{\text {water }} / \eta_{n} \operatorname{coth}\left(q H_{\text {water }}\right)\right]^{2}} .
$$

Here, $I_{0}$ and $I_{1}$ are modified Bessel functions, and $B=$ $N \omega_{v}^{2} /\left(32 \pi^{3} \mathcal{A}\right)$ is a prefactor related to the total enstrophy.

To discuss the scaling regimes predicted from Eq. (1), we consider a simplified situation with just one external fluid layer, either above or below the active film. This simpler setup is enough to capture the effects of external fluid layers on the active film. The energy spectrum then depends on three length scales: the mean vortex radius $R_{*}$, the layer

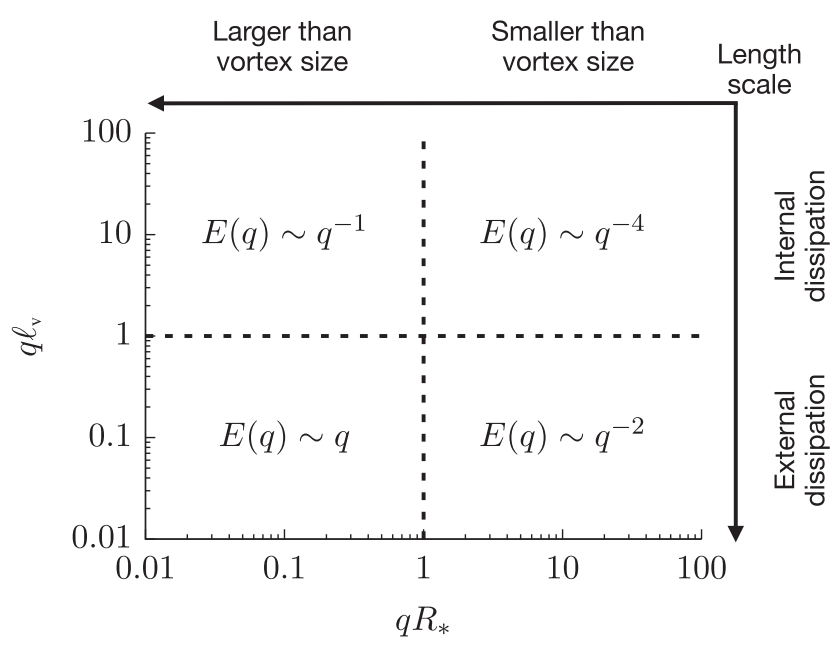

FIG. 2. Scaling regimes of turbulent flows in an active nematic film in contact with an external fluid. The different regimes are predicted at length scales $(\sim 1 / q)$ either larger or smaller than the mean vortex radius $R_{*}$, the viscous length $\ell_{v}=\eta_{n} / \eta_{\text {ext }}$, and the thickness $H$ of the external fluid layer. This figure summarizes the scalings in the thick-layer limit $q H \gg 1$; see Fig. 6 for the predictions in the thin-layer limit $q H \ll 1$. 
thickness $H$, and the viscous length $\ell_{v}=\eta_{n} / \eta_{\text {ext }}$ defined by the ratio of the two-dimensional viscosity of the nematic film $\eta_{n}$ and the three-dimensional viscosity of the external fluid $\eta_{\text {ext }}$. In the thick-layer limit, $q H \gg 1$, we summarize the predicted scaling regimes in Fig. 2, which we discuss below. The thin-layer limit, $q H \ll 1$, is discussed in Fig. 6 in Appendix B 3.

Active flows are characterized by different scaling laws at scales smaller and larger than the mean vortex size $R_{*}$ (horizontal axis in Fig. 2). Furthermore, at length scales smaller than the viscous length, $\ell_{v}=\eta_{n} / \eta_{\text {ext }}$, dissipation is dominated by the viscosity of the active film. As a result, in this regime, the scaling laws are those recently predicted and numerically demonstrated for isolated active nematic films [36,37], with no effect of the external fluid (top half in Fig. 2). At length scales larger than $\ell_{v}$, however, dissipation in the external fluid dominates, yielding new scaling laws (bottom half in Fig. 2).

\section{COMPARISON TO EXPERIMENTS}

As described later, by fitting the theory to the experimental measurements, we infer the active film viscosity $\eta_{n} \approx$ $4 \mathrm{Pas} \mu \mathrm{m}$. Therefore, using $\eta_{\text {water }}=1 \mathrm{mPas}$ we obtain the water viscous length $\ell_{\text {water }}=\eta_{n} / \eta_{\text {water }} \approx 4 \mathrm{~mm}$, which is larger than the largest length scale of our measurements, $q_{\min }^{-1} \sim 0.3 \mathrm{~mm}$. Hence, we have $q \ell_{\text {water }} \gg 1$ for all $q$ values. Therefore, the flows in the water layer do not produce any new scaling regimes in our experiments. In contrast, varying oil viscosity over orders of magnitude, we reach oil viscous lengths $\ell_{\text {oil }}=\eta_{n} / \eta_{\text {oil }}$ that fall within our measurement window. Consequently, the flows in the oil layer are

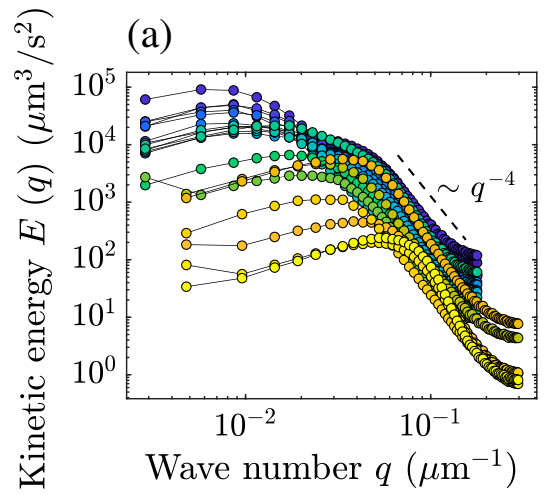

(c)

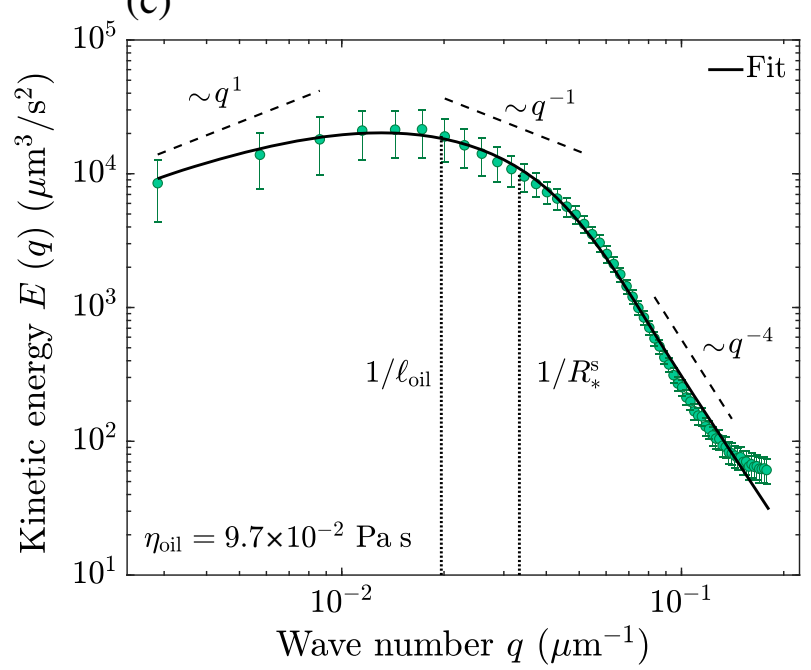

(b)

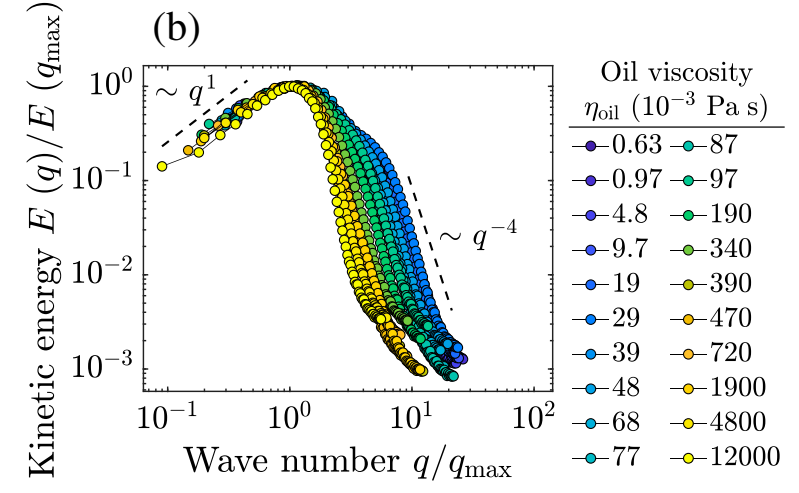

(d)

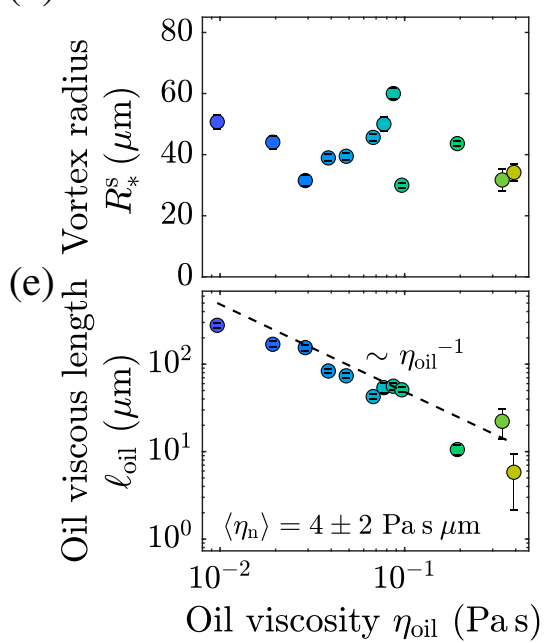

FIG. 3. Oil viscosity tunes the scaling regimes of active nematic turbulence. (a) Kinetic energy spectra of turbulent flows in an active nematic film in contact with a layer of oil, for 20 different oil viscosities. The data are averaged over 500 frames. (b) Rescaling each spectrum by its maximum and its corresponding wave number clearly showcases the large-scale scaling regime. (c) Fit of Eq. (1) to a representative spectrum at intermediate oil viscosity (see fits for all oil viscosities in Fig. S1 in Supplemental Material [52]). As predicted by our theory (see Fig. 2), the spectrum features signatures of three scaling regimes, separated by two crossover lengths: the mean vortex size $R_{*}^{s}$ and the viscous length $\ell_{\text {oil }}=\eta_{n} / \eta_{\text {oil }}$ (vertical dashed lines). Averages are over 500 frames. Error bars are standard deviations. (d),(e) Mean vortex radius (d) and oil viscous length (e) obtained from the spectral fits in the range of intermediate oil viscosities in which the theory fits the data well. Error bars are standard errors of the mean. The mean nematic viscosity obtained from these fits is indicated in (e) as an inset (see also Fig. S2 [52]). 
responsible for some of the scaling regimes that we observe. Our measurements probe length scales that are smaller than the oil layer thickness $H_{\text {oil }} \approx 3 \mathrm{~mm}$. Thus, our experiments operate in the thick-layer limit, with scaling properties as predicted in Fig. 2.

Consistent with these predictions, we experimentally observe $E(q) \sim q^{-4}$ at small scales, and $E(q) \sim q^{1}$ at large scales, for all oil viscosities [Figs. 3(a) and 3(b)]. For low and intermediate oil viscosities, we also observe signatures of the $E(q) \sim q^{-1}$ regime at intermediate length scales, larger than the vortex size $R_{*}$ but smaller than the viscous length $\ell_{\text {oil }}$ [Fig. 3(c)]. In our measurements, the scaling regimes are limited in extension, and the data alone do not provide conclusive evidence for the scaling laws. However, the agreement with the theoretical predictions confirms that the active turbulent flows in our system indeed follow underlying scaling laws.

The $q^{-4}$ and $q^{-1}$ scaling behaviors are intrinsic properties of an active nematic film, respectively characterizing the small-scale flows inside vortices and the large-scale flows due to hydrodynamic interactions in the film [37]. In contrast, the $q^{1}$ scaling stems from the hydrodynamic coupling to an external fluid. All these scaling relations involve universal exponents, which are independent of the properties of the fluids. Consistently, by varying the oil viscosity, we tune the range of the different regimes without changing their scaling exponents. In other words, the values of the exponents are properties of the equations of active nematics and not of their parameters. The range of each scaling regime, however, depends on parameters.

Having demonstrated the scaling regimes, we quantitatively compare our prediction for the full energy spectrum [Eq. (1)] to the experimental data. Knowing the values of $\eta_{\text {water }}, \eta_{\text {oil }}, H_{\text {water }}$, and $H_{\text {oil }}$, we use $B, \eta_{n}$, and $R_{*}$ in Eq. (1) as fitting parameters. Despite its assumptions, our theory fits the data remarkably well for a wide range of intermediate oil viscosities $\left(9.7 \times 10^{-3}<\eta_{\text {oil }}<0.39 \mathrm{Pas}\right.$; see Fig. S1 in Supplemental Material [52]), as exemplified in Fig. 3(c). Visual inspection of the experiments suggests smaller vortices as $\eta_{\text {oil }}$ increases (see insets in Fig. 1 and Movie 2 [52]). Yet, the mean vortex radius obtained from these fits, $R_{*}^{s}$, is independent of oil viscosity [Fig. 3(d)]. Finally, the oil viscous length $\ell_{\text {oil }}=\eta_{n} / \eta_{\text {oil }}$ decreases as $\sim 1 / \eta_{\text {oil }}$ [Fig. 3(e)], indicating that $\eta_{n}$ does not vary with oil viscosity in the range of validity of the model (Fig. S2 [52]). Hence, the fits allow us to estimate the viscosity of the active nematic: $\left\langle\eta_{n}\right\rangle=4 \pm 2 \operatorname{Pas} \mu \mathrm{m}$. This value is 2 orders of magnitude smaller than that obtained by Guillamat et al. from the speed of topological defects [56], possibly due to the overestimation of the flow screening length in their model. Our estimate, however, is of the same order as the value obtained by Rivas et al. from the flow profile around vortices [61].

\section{VORTEX SIZE SELECTION AND CORRELATIONS}

To try to rationalize the fact that $R_{*}^{s}$ is independent of oil viscosity, we perform a linear stability analysis. Because of the coupling to the external fluid, the linear growth rate of the spontaneous-flow instability that powers active nematic turbulence acquires a maximum at finite wavelengths [53-55] [Appendix C and Fig. 7(a)]. This maximum selects a characteristic scale $\lambda_{m}$ of flow patterns at the onset of turbulence [24]. Does $\lambda_{m}$ also determine vortex size in fully developed turbulence? The linear analysis predicts that $\lambda_{m}$ changes with oil viscosity [Fig. 7(b)], in contrast with experimental observations for $R_{*}$. Therefore, $\lambda_{m}$ does not seem to directly determine vortex size in the turbulent state, as $\lambda_{m}$ and $R_{*}^{s}$ have different dependencies on oil viscosity.

Nonlinear effects may modify the scale $\lambda_{m}$ selected by the linear dynamics upon the instability. In fact, in stationary fully developed turbulence, earlier work has shown that vortex size is determined by the nonlinear dynamics of the active nematic and given by the critical wavelength of the instability $\lambda_{c}$ [37]. Here, we show theoretically that this length scale $\lambda_{c}$ is largely independent of oil viscosity [Appendix C and Fig. 7(c)], consistent with the behavior $R_{*}^{s}$ with oil viscosity [Fig. 3(d)]. Therefore, $\lambda_{c}$ could determine vortex size in the turbulent state.

To further investigate this issue experimentally, we directly measure the distributions of vortex areas $n(a)$ [21,23,29] [Fig. 4(a), Appendix A]. Fitting their exponential tails as $n(a) \propto \exp \left(-a / a^{*}\right)$ [36], we obtain a mean vortex radius $R_{*}^{v}=\sqrt{a_{*} / \pi}$ as a function of oil viscosity [Fig. 4(b)]. In Fig. S3 in Supplemental Material [52] we compare $R_{*}^{v}$ and $R_{*}^{s}$ in the range of validity of our spectral fits. We find that both methods yield very similar values of vortex radius, which is roughly independent of oil viscosity, again suggesting that nonlinear effects contribute to vortex size selection in our system. Yet, for high oil viscosities, outside the range of validity of our spectral fits, we find that $R_{*}^{v}$ decreases with oil viscosity [Fig. 4(b)]. Explaining these results will require further research on vortex size selection in active turbulence.

Finally, we use our measurements to examine some of the assumptions of the theoretical framework. We observe that vortex area distributions have exponential tails [Fig. 4 (a)], in agreement with both the theoretical assumption and previous experiments $[21,23,29]$. Here, we find that this feature does not change with the oil viscosity. We also measure the correlation functions for velocity and vorticity $[41,62,63]$ [Figs. 4(c) and 4(d)] and obtain the corresponding correlation lengths [insets in Figs. 4(c) and 4(d)], which exhibit dependencies on the oil viscosity that are very similar to that of the vortex size $R_{*}^{v}$ [Fig. 4(b)]. This observation suggests that these lengths are all proportional to one another (Fig. S4 [52]), validating our theoretical 
(a)

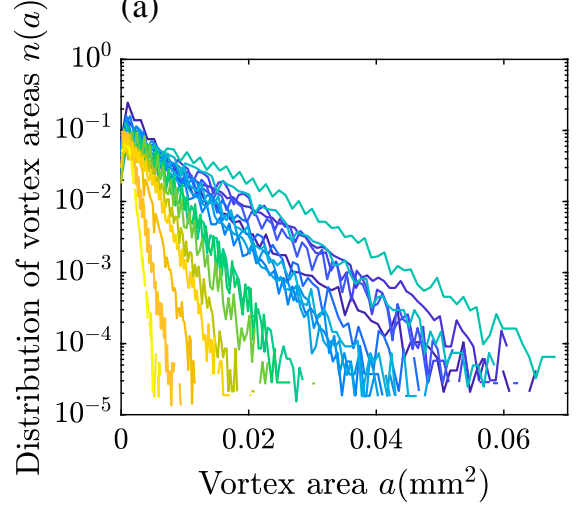

(c)

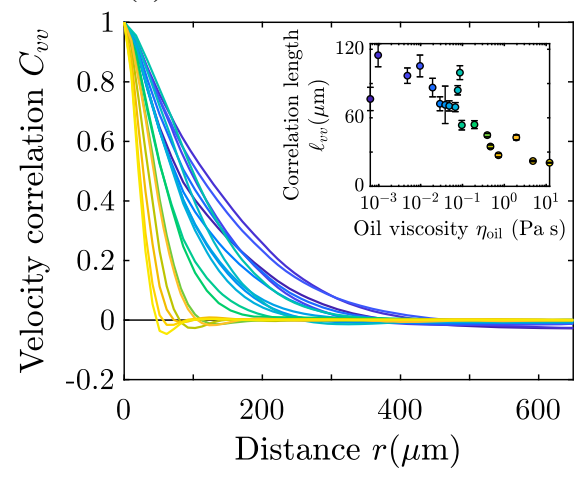

(b)

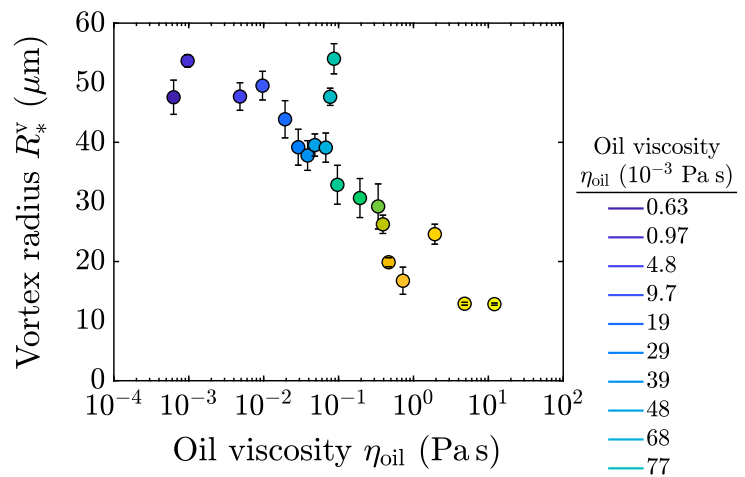

(d)

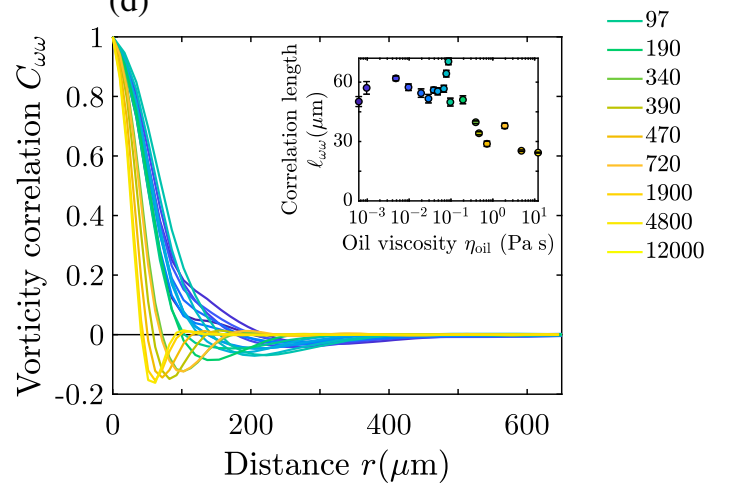

FIG. 4. Vortex size and correlation functions in active nematic turbulence. (a) Vortex area distributions in the active turbulence regime for 20 different oil viscosities. The distributions are obtained by measuring vortices in 500 frames. (b) Mean vortex radius obtained from the exponential tails of the vortex area distributions in (a) (see Appendix A). Error bars are standard errors of the mean. (c),(d) Spatial autocorrelation functions of the velocity (c) and vorticity (d) fields, for all 20 oil viscosities. The data are averaged over 500 frames. The insets show the corresponding correlation lengths, defined by the conditions $C_{v v}\left(\ell_{v v}\right)=0.5$ and $C_{\omega \omega}\left(\ell_{\omega \omega}\right)=0.5$, respectively. Error bars are standard errors of the mean.

assumption that there is a unique scale related to activity $\left(R_{*}\right)$. However, at intermediate and high oil viscosities, we measure negative correlations of the vorticity field at distances comparable to, and even larger than, the vortex size [Fig. 4(d)]. Thus, our data call for future work on the theory of active turbulence, going beyond mean-field approximations and accounting for vortex-vortex correlations. Other improvements to the theory could take into account the structure of defect cores, and include a more accurate treatment of flow-alignment effects.

\section{CONCLUSION}

In summary, we have experimentally measured and theoretically explained universal scaling laws in active turbulence, thus drawing parallels to classical turbulence. Specifically, we have found scaling regimes that are intrinsic to an active nematic film as well as other regimes that result from the coupling to an external fluid. In addition, we have developed a theoretical framework that provides the rationale for the different regimes and yields an explicit form for the turbulent spectra as a function of parameters. By fitting the predictions to the data, we have extracted the crossover length scales and shown how they depend on the viscosity of the external fluid. Our analysis of these results paves the way toward addressing open questions in active turbulence, from vortex size selection to the role of vortex-vortex correlations, and to pursue a deeper understanding of the fundamental similarities and differences between inertial and active turbulence.

\section{ACKNOWLEDGMENTS}

The authors are indebted to the Brandeis University MRSEC Biosynthesis facility for providing the tubulin. We thank M. Pons, A. LeRoux, and G. Iruela (Universitat de Barcelona) for their assistance in the expression of motor proteins. B. M.-P., J. I.-M., and F. S. acknowledge funding from MINECO (Projects No. FIS2016-78507-C2-1-P, AEI/ FEDER, EU, and No. PID2019-108842 GB-C22). J.C. acknowledges support from MINECO (Projects No. FIS2016-78507-C2-2-P, AEI/FEDER, EU, and No. PID2019-108842 GB-C21) and Generalitat de Catalunya under Project No. 2017-SGR-1061. B. M.-P. acknowledges funding from Generalitat de Catalunya through a FI-2018 Ph.D. Fellowship. Brandeis 
University MRSEC Biosynthesis facility is supported by NSF MRSEC DMR-2011846. R. A. acknowledges support from the Human Frontier Science Program (LT000475/ 2018-C). F. M. and R. G. acknowledge support from the Max Planck Society. R. A. and R. G. acknowledges discussions with the participants of the virtual "Active 20" KITP program, supported in part by the National Science Foundation under Grant No. NSF PHY-1748958.

\section{APPENDIX A: METHODS}

\section{Preparation of the active nematic}

Clusters of biotinylated kinesin-1 (K401-BCCP-6His) are assembled with tetrametric streptavidin at a $\sim 2: 1$ ratio. Afterward, these molecular motors are mixed with adenosine triphosphate (ATP) and an ATP-regenerating system [pyruvate kinase/lactic dehydrogenase enzymes (PK/LDH) and phosphoenol pyruvate (PEP)]. The nonadsorbing polymer polyethylene glycol (PEG) $(20 \mathrm{kDa})$ is used as depleting agent. To avoid photobleaching and protein oxidation, a mixture of antioxidants [glucose, catalase, 1,4-dithiothreitol (DTT), trolox, and glucose oxidase] is also included. Biocompatibility of the active nematic layer with the oil interface is assured with the surfactant pluronic F-127. This final suspension is mixed with $\sim 1.5 \mu \mathrm{m}$ microtubules stabilized with guanosine-5'-[( $\alpha, \beta)$-methyleno]triphosphate (GMPCPP) (Jena Bioscience, NU-405S), of which the $0.8 \%$ is labeled with the Alexa-647 fluorophore. The scattered fluorescent microtubules form a speckle pattern (see Movie 1 in Supplemental Material [52]), from which we measure the velocity field through particle image velocimetry (PIV). Final compound concentrations are listed in Table I.

TABLE I. Composition of the active nematic. (Definitions of acronyms found in text.).

\begin{tabular}{lc}
\hline \multicolumn{2}{c}{ Active nematic composition } \\
\hline Compound & Concentration \\
\hline Streptavidin & $0.16 \mu \mathrm{M}$ \\
Kinesin & $0.32 \mu \mathrm{M}$ \\
DTT & $5.8 \mathrm{mM}$ \\
PEG $(20 \mathrm{kDa})$ & $1.6 \% \mathrm{w} / \mathrm{w}$ \\
PEP & $27 \mathrm{mM}$ \\
Trolox & $2.1 \mathrm{mM}$ \\
MgCl & \\
ATP & $3.3 \mathrm{mM}$ \\
PK/LDH & $1.5 \mathrm{mM}$ \\
Pluronic & $27 \mathrm{IU} / \mathrm{mL}$ \\
Glucose & $0.44 \%(\mathrm{w} / \mathrm{w})$ \\
Catalase & $3.4 \mathrm{mg} / \mathrm{mL}$ \\
Glucose oxidase & $0.040 \mathrm{mg} / \mathrm{mL}$ \\
Microtubules & $0.23 \mathrm{mg} / \mathrm{mL}$ \\
\hline \hline
\end{tabular}

The active nematic (AN) is finally assembled by depositing a $1.5 \mu \mathrm{L}$ droplet onto a polyacrylamide-functionalized glass slide within a $10 \mathrm{~mm}$ wide and $10 \mathrm{~mm}$ high polypropylene cylinder, and covered with $300 \mu \mathrm{L}$ of polydimethylsiloxane oil (PDMS) with a viscosity in the range of $6.3 \times 10^{-4}$ to $12 \mathrm{~Pa}$ s. Micotubules spontaneously adsorb onto the oil-water interface leading to the formation of the AN. The mean height of the water layer is obtained considering a cylinder and measuring the cross-section area of the drop through fluorescence microscopy images. In the case of the oil layer, its height is directly measured with a millimetric ruler. We take the height at the center, where the AN drop is placed, neglecting the meniscus.

To have intermediate oil viscosities, we prepare oil mixtures. The final viscosity is estimated using the Arrhenius mixing rule $\log \left(\eta_{12}\right)=x_{1} \log \left(\eta_{1}\right)+\left(1-x_{1}\right) \log \left(\eta_{2}\right)$, where $\eta_{12}, \eta_{1}$, and $\eta_{2}$ are the oil viscosities of the oil mixture and of the mixed compounds 1 and 2, respectively, and $x_{1}$ is the molar fraction of oil $1[64,65]$.

\section{Observation of the active nematic}

The active nematic layer is imaged by means of fluorescence microscopy (Nikon Eclipse Ti2-U) with an Andor Zyla 4.2 Plus camera controlled with the opensource software ImageJ Micro-Manager [66]. As light source, we use a red LED coupled to a Cy5 cube filter. Images are acquired typically at a frame rate of $2 \mathrm{~Hz}$ and with a spatial resolution of $2.14 \mu \mathrm{m} / \mathrm{pixel}$. In the case of experiments with high oil viscosities ( $>1 \mathrm{Pas}$ ) the frame rate is decreased to $1 \mathrm{~Hz}$ and the spatial resolution is increased to $1.28 \mu \mathrm{m} /$ pixel.

\section{Flow field measurements}

Raw images are treated with the open source software ImageJ [67]. In general, light intensity is equalized by dividing the intensity of each frame by the time average of the sequence. Further noise removal is achieved with a mean filter with a width of 2-4 pixels. Afterward, the velocity field is computed with PIVlab for MATLAB [68]. PIV window size is set as 1/64 of the lateral system size. To reduce noise, we use the option $5 x$ repeated correlation. The velocity field is finally smoothed using the smoothn function developed by Garcia [69].

\section{Kinetic energy spectrum}

The kinetic energy per unit mass is defined as

$$
E=\frac{1}{2}\left\langle\int_{L^{2}} \boldsymbol{v}^{2} d^{2} \boldsymbol{r}\right\rangle
$$

where $\langle\cdot\rangle$ indicates a time average and $L^{2}$ is the system size. Introducing the Fourier decomposition of the velocity field $\boldsymbol{v}(\vec{r}, t)=\sum_{q} \tilde{\tilde{\boldsymbol{v}}}(\boldsymbol{q}) e^{i \boldsymbol{q} \cdot \boldsymbol{r}}$, we obtain 


$$
E=\frac{1}{2} L^{2}(2 \pi)^{2} \sum_{\boldsymbol{q}}\left\langle|\tilde{\tilde{\boldsymbol{v}}}(\boldsymbol{q})|^{2}\right\rangle
$$

where $\boldsymbol{q}=2 \pi / L\left(n_{x}, n_{y}\right)$, with $n_{x}, n_{y} \in \mathbb{N}$. Now, we define the spectral density of the kinetic energy $E(\boldsymbol{q})$ as

$$
\frac{E}{L^{2}}=\sum_{q_{x}} \sum_{q_{y}} E(\boldsymbol{q}) \Delta q_{x} \Delta q_{y}=\frac{(2 \pi)^{2}}{L^{2}} \sum_{q_{x}} \sum_{q_{y}} E(\boldsymbol{q}) .
$$

Combining Eq. (A2) with Eq. (A3), we obtain

$$
E(\boldsymbol{q})=\frac{L^{2}}{2}\left\langle|\tilde{\boldsymbol{v}}(\boldsymbol{q})|^{2}\right\rangle
$$

We consider the overall system to be isotropic and, hence, we can angle average $E(\boldsymbol{q})$ to get $E(q)$ in terms of the module of the wave number $q=|\boldsymbol{q}|$, and we get

$$
E(q)=\frac{L^{2}}{2} q \sum_{\varphi}\left\langle|\tilde{\tilde{v}}(q, \varphi)|^{2}\right\rangle \Delta \varphi
$$

where $\varphi$ is the azimuth of the wave vector $\boldsymbol{q}$. Note that in experiments we use the discrete Fourier transform while in the theory we use the continuous Fourier transform. This explains the difference in the prefactors between Eq. (A5) and Eq. (B15). For each experiment, we average a total of 500 frames.

Afterward, we fit Eq. (1) to the experimental $E(q)$ with Mathematica v10.

\section{Velocity and vorticity correlations}

We compute the velocity and vorticity correlation functions ( $C_{v v}$ and $C_{\omega \omega}$, respectively) using the WienerKhinchin theorem: $C_{x x}(\mathbf{r})=\mathcal{F}^{-1}\left[|\tilde{\mathbf{x}}(\mathbf{q})|^{2}\right]$, where $\mathcal{F}^{-1}[\cdot]$ denotes the inverse Fourier transform operator and $\tilde{x}$ denotes either $\boldsymbol{v}$ or $\omega$.

\section{Exponential distribution of the vortex areas and mean vortex radius}

The flows created by our active system clearly feature eddies (see Movies 1 and 2 in Supplemental Material [52]) that we identify and characterize using the Okubo-Weiss (OW) parameter, as described previously $[21,23,36]$. Briefly, $\mathrm{OW}$ is calculated as $\mathrm{OW}=\left(\partial_{x} v_{x}+\partial_{y} v_{y}\right)^{2}-$ $4\left(\partial_{x} v_{x}\right)\left(\partial_{y} v_{y}\right)+4\left(\partial_{x} v_{y}\right)\left(\partial_{y} v_{x}\right)$. Regions with OW $<0$ are good vortex candidates. To determine whether such regions are vortices, we check if they feature a singularity by computing the winding number $W(r)=$ $1 / 2 \pi \oint_{0}^{2 \pi} \operatorname{atan}\left[v_{y}(r, \varphi) / v_{x}(r, \varphi)\right] d \varphi$ of the velocity field at a distance $r=24$ pixels from their center. If a region with OW $<0$ has a winding number $W \in[0.95,1.05]$, it is accepted as a vortex. The total area of each swirl is determined by the connected area with $\mathrm{OW}<0$. This vortex locator algorithm allows us to extract a distribution of vortex areas $n(a)=N(a) / \sum_{a} N(a)$, where $N(a)$ is the number of vortices with area $a$. Since $n(a)$ follows an exponential distribution, we extract a characteristic vortex area $a_{*}$ and radius $R_{*}^{v}=\sqrt{a_{*} / \pi}$.

\section{APPENDIX B: HYDRODYNAMIC THEORY OF ACTIVE TURBULENCE WITH EXTERNAL DISSIPATION}

\section{Hydrodynamic Green's function}

In our experimental setup, a thin film of the active nematic fluid is in contact with a thicker layer of water underneath, and a much thicker layer of oil above. The water layer is supported by a solid substrate, and the oil layer is in contact with air (Fig. 5). Active flows in the nematic film induce flows in both passive fluid layers, which in turn influence the flows in the active film. To account for this hydrodynamic coupling, here we obtain the Green function for the flow field in a two-dimensional viscous fluid film in contact with three-dimensional layers of other viscous fluids. This calculation generalizes previous work [60] by considering the simultaneous hydrodynamic coupling to two fluid layers with different boundary conditions, as in our experimental setup (Fig. 5).

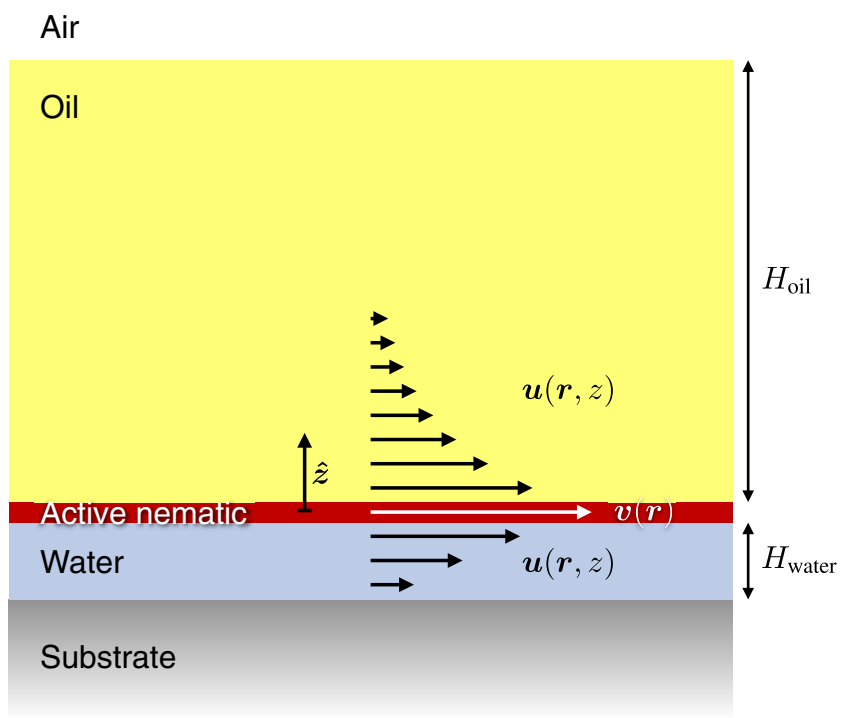

FIG. 5. Schematic of the experimental setup and flow fields (side view). The thicknesses of the fluid layers are not to scale. The actual thickness of the active nematic film is $h \approx 2 \mu \mathrm{m}$, whereas the thicknesses of the passive fluid layers are $H_{\text {water }} \approx$ $40 \mu \mathrm{m}$ and $H_{\text {oil }} \approx 3 \mathrm{~mm}$. We treat the active nematic as a twodimensional film. White and black arrows represent the flow fields in the active nematic film and in the passive fluid layers, respectively. The flow is planar and it penetrates into the oil and water layers. Here, we represent flow penetration according to Eq. (B9) for a planar wave number $q /(2 \pi)=5 \times 10^{-3} \mu \mathrm{m}^{-1}$, which lies in the range of our experimental measurements. 
All flows in our system take place at very low Reynolds numbers. Therefore, inertial forces are negligible (Stokes limit), and momentum conservation reduces to force balance. For the active nematic film, with two-dimensional shear viscosity $\eta_{n}$ and incompressible flow field $\boldsymbol{v}(\boldsymbol{r})$, force balance can be written as

$$
\eta_{n} \nabla^{2} \boldsymbol{v}-\boldsymbol{\nabla} P+\boldsymbol{f}_{\text {water }}+\boldsymbol{f}_{\text {oil }}+\boldsymbol{f}=\mathbf{0} .
$$

Here, the first term accounts for the viscous stress within the nematic film, $P$ is the film's two-dimensional pressure, and $f_{\text {water }}$ and $\boldsymbol{f}_{\text {oil }}$ are the viscous force densities exerted by the water and oil layers, respectively, on the active fluid film. Finally, $f$ is the remaining force surface density acting on the film. In our system, this force density results from stresses associated with the orientational order of the nematic film, including elastic, flow-alignment, and active stresses. Here, we derive the Green function treating $f$ as a source, without specifying its expression. Rather, we express the flow field of the nematic film in terms of the source force $f$ as

$$
v_{\alpha}(\boldsymbol{r})=\int G_{\alpha \beta}\left(\boldsymbol{r}-\boldsymbol{r}^{\prime}\right) f_{\beta}\left(\boldsymbol{r}^{\prime}\right) d \boldsymbol{r}^{\prime},
$$

where $G_{\alpha \beta}\left(\boldsymbol{r}-\boldsymbol{r}^{\prime}\right)$ is a hydrodynamic Green function, namely, a generalization of Oseen's tensor. Greek indices indicate spatial components, and summation over repeated indices is implicit. In Fourier space, we have

$$
\tilde{v}_{\alpha}(\boldsymbol{q})=\tilde{G}_{\alpha \beta}(\boldsymbol{q}) \tilde{f}_{\beta}(\boldsymbol{q}),
$$

where we have introduced the Fourier decomposition as

$$
\boldsymbol{v}(\boldsymbol{r})=\int \frac{d^{2} \boldsymbol{q}}{(2 \pi)^{2}} \tilde{\boldsymbol{v}}(\boldsymbol{q}) e^{i \boldsymbol{q} \cdot \boldsymbol{r}} .
$$

To obtain the Green function, we must obtain the viscous stress exerted by the water and oil layers on the active fluid film,

$$
\begin{gathered}
\boldsymbol{f}_{\text {water }}(\boldsymbol{r})=-\left.\eta_{\text {water }} \frac{\partial \boldsymbol{u}_{\|}(\boldsymbol{r}, z)}{\partial z}\right|_{z=0^{-}}, \\
\boldsymbol{f}_{\text {oil }}(\boldsymbol{r})=\left.\eta_{\text {oil }} \frac{\partial \boldsymbol{u}_{\|}(\boldsymbol{r}, z)}{\partial z}\right|_{z=0^{+}},
\end{gathered}
$$

respectively. Here, $\boldsymbol{u}(\boldsymbol{r}, z)$ is the three-dimensional flow field of the passive fluids, and the subscript $\|$ indicates the components along the active film's plane $(z=0$; see Fig. 5). The viscous flows in the passive layers obey the Stokes equation,

$$
\eta_{\text {water }} \nabla^{2} \boldsymbol{u}-\boldsymbol{\nabla} p=\mathbf{0}, \quad-H_{\text {water }}<z<0,
$$

$$
\eta_{\mathrm{oil}} \nabla^{2} \boldsymbol{u}-\boldsymbol{\nabla} p=\mathbf{0}, \quad 0<z<H_{\mathrm{oil}},
$$

with $p$ the three-dimensional pressure. These flows are driven by the hydrodynamic coupling with the active film: $\boldsymbol{u}_{\|}\left(\boldsymbol{r}, 0^{+}\right)=\boldsymbol{u}_{\|}\left(\boldsymbol{r}, 0^{-}\right)=\boldsymbol{v}(\boldsymbol{r})$. As shown in Refs. [58,59], if the film's flow is incompressible, $\boldsymbol{\nabla} \cdot \boldsymbol{v}=0$, then the pressure in the fluid layers is uniform, $\boldsymbol{\nabla} p=\mathbf{0}$, and the out-of-plane component of the velocity vanishes everywhere, $u_{z}=0$. Therefore, the layers' flow field is planar and harmonic; it obeys Laplace's equation:

$$
\begin{array}{cc}
\eta_{\text {water }} \nabla^{2} \boldsymbol{u}_{\|}=\mathbf{0}, & -H_{\text {water }}<z<0, \\
\eta_{\text {oil }} \nabla^{2} \boldsymbol{u}_{\|}=\mathbf{0}, & 0<z<H_{\text {oil }} .
\end{array}
$$

To obtain the Green function in Fourier space, as in Eq. (B3), we solve Eq. (B7) in terms of the planar Fourier modes of the flow field, $\tilde{\boldsymbol{u}}(\boldsymbol{q}, z)$, which obey

$$
\begin{array}{r}
\eta_{\text {water }}\left(\partial_{z}^{2}-q^{2}\right) \tilde{\boldsymbol{u}}=\mathbf{0}, \quad-H_{\text {water }}<z<0, \\
\eta_{\text {oil }}\left(\partial_{z}^{2}-q^{2}\right) \tilde{\boldsymbol{u}}=\mathbf{0}, \quad 0<z<H_{\text {oil }} .
\end{array}
$$

The water layer is in contact with a solid substrate at $z=-H_{\text {water }}$, where we assume a no-slip boundary condition: $\boldsymbol{u}_{\|}\left(\boldsymbol{r},-H_{\text {water }}\right)=\mathbf{0}$. Respectively, the oil layer is in contact with air at $z=H_{\text {oil }}$, where we assume a no-shearstress boundary condition: $\left.\partial_{z} \boldsymbol{u}_{\|}(\boldsymbol{r}, z)\right|_{z=H_{\text {oil }}}=0$. With these boundary conditions and $\boldsymbol{u}_{\|}\left(\boldsymbol{r}, 0^{+}\right)=\boldsymbol{u}_{\|}\left(\boldsymbol{r}, 0^{-}\right)=\boldsymbol{v}(\boldsymbol{r})$, the solutions to Eq. (B8) are

$$
\begin{aligned}
\tilde{\boldsymbol{u}}_{\|}(\boldsymbol{q}, z)= & {\left[\cosh (q z)+\operatorname{coth}\left(q H_{\text {water }}\right) \sinh (q z)\right] \tilde{\boldsymbol{v}}(\boldsymbol{q}), } \\
& -H_{\text {water }}<z<0,
\end{aligned}
$$

$\tilde{\boldsymbol{u}}_{\|}(\boldsymbol{q}, z)=\left[\cosh (q z)-\tanh \left(q H_{\mathrm{oil}}\right) \sinh (q z)\right] \tilde{\boldsymbol{v}}(\boldsymbol{q})$,

$$
0<z<H_{\text {oil }} \text {. }
$$

These solutions show that the flow penetrates into the oil and water layers to depths given by the inverse of the inplane wave number, $1 / q$, unless limited by the layers' thicknesses, $H_{\text {water }}$ and $H_{\text {oil }}$, as illustrated in Fig. 5. Introducing Eq. (B9) into Eq. (B5), we obtain

$$
\begin{array}{r}
\tilde{\boldsymbol{f}}_{\text {water }}(\boldsymbol{q})=-\eta_{\text {water }} q \operatorname{coth}\left(q H_{\text {water }}\right) \tilde{\boldsymbol{v}}(\boldsymbol{q}), \\
\tilde{\boldsymbol{f}}_{\text {oil }}(\boldsymbol{q})=-\eta_{\text {oil }} q \tanh \left(q H_{\text {oil }}\right) \tilde{\boldsymbol{v}}(\boldsymbol{q}) .
\end{array}
$$

Finally, introducing these results into the Fourier transform of Eq. (B1), and using the incompressibility condition $\boldsymbol{q} \cdot \tilde{\boldsymbol{v}}=0$, we obtain the Green function: 


$$
\tilde{G}_{\alpha \beta}(\boldsymbol{q})=\frac{\delta_{\alpha \beta}-q_{\alpha} q_{\beta} / q^{2}}{\eta_{n} q^{2}+\eta_{\mathrm{oil}} q \tanh \left(q H_{\mathrm{oil}}\right)+\eta_{\mathrm{water}} q \operatorname{coth}\left(q H_{\mathrm{water}}\right)} .
$$

This function describes the hydrodynamic interactions in the active nematic film, accounting both for the direct interactions due to its incompressibility and viscosity, and also for the indirect interactions mediated by the oil and water layers. The ratios between the viscosity of these external fluids and the two-dimensional viscosity of the nematic film define two viscous length scales:

$$
\ell_{\mathrm{oil}}=\eta_{n} / \eta_{\mathrm{oil}}, \quad \ell_{\mathrm{water}}=\eta_{n} / \eta_{\text {water }}
$$

At scales larger than this viscous length, dissipation in the external fluid layer (either oil or water) dominates over dissipation within the nematic film.

\section{Kinetic energy spectrum}

Here, we derive an analytical expression for the kinetic energy spectrum of the turbulent flows in our system. To this end, we establish a relationship between the flow field of our coupled three-fluid system (Fig. 5) and the vorticity field of an isolated active nematic film. By means of this relationship, we combine the hydrodynamic Green function obtained in Appendix B 1 with Giomi's mean-field theory of active nematic turbulence [36] to predict the flow power spectrum in our experimental system.

The kinetic energy per unit mass density $E$ of the active nematic flows is given by

$$
E=\frac{1}{2} \int \boldsymbol{v}^{2} d^{2} \boldsymbol{r}
$$

Using the Fourier modes $\tilde{\boldsymbol{v}}(\boldsymbol{q})$ of the flow field, as introduced in Eq. (B4), the angle-averaged spectrum $E(q)$, with $q=|\boldsymbol{q}|$ the wave number, is defined by

$$
\langle E\rangle=\frac{1}{2} \int \frac{d^{2} \boldsymbol{q}}{(2 \pi)^{2}}\left\langle|\tilde{\boldsymbol{v}}(\boldsymbol{q})|^{2}\right\rangle=\mathcal{A} \int_{0}^{\infty} E(q) d q,
$$

where $\mathcal{A}$ is the area of the system and $\langle\cdot\rangle$ averages over realizations. In states where correlations of the flow field are isotropic, $E(q)$ is given by

$$
E(q)=\frac{1}{4 \pi \mathcal{A}} q\left\langle|\tilde{\boldsymbol{v}}(\boldsymbol{q})|^{2}\right\rangle .
$$

To obtain the velocity power spectrum, we use Eqs. (B3) and (B11), which give

$$
\begin{aligned}
\left\langle|\tilde{\boldsymbol{v}}(\boldsymbol{q})|^{2}\right\rangle & =\left(\delta_{\alpha \beta}-\frac{q_{\alpha} q_{\beta}}{q^{2}}\right)\left(\delta_{\alpha \gamma}-\frac{q_{\alpha} q_{\gamma}}{q^{2}}\right) \frac{\left\langle\tilde{f}_{\beta}(\boldsymbol{q}) \tilde{f}_{\gamma}^{*}(\boldsymbol{q})\right\rangle}{\Lambda^{2}(q)} \\
& =\frac{1}{\Lambda^{2}(q)}\left\langle|\tilde{\boldsymbol{f}}(\boldsymbol{q})|^{2}-\frac{q_{\alpha} q_{\beta}}{q^{2}} \tilde{f}_{\alpha}(\boldsymbol{q}) \tilde{f}_{\beta}^{*}(\boldsymbol{q})\right\rangle \\
& =\frac{1}{q^{2} \Lambda^{2}(q)}\left\langle q_{y}^{2}\left|\tilde{f}_{x}\right|^{2}+q_{x}^{2}\left|\tilde{f}_{y}\right|^{2}-q_{x} q_{y}\left(\tilde{f}_{x} \tilde{f}_{y}^{*}+\tilde{f}_{y} \tilde{f}_{x}^{*}\right)\right\rangle .
\end{aligned}
$$

Here, we have introduced the notation

$\Lambda(q) \equiv \eta_{n} q^{2}+\eta_{\text {oil }} q \tanh \left(q H_{\text {oil }}\right)+\eta_{\text {water }} q \operatorname{coth}\left(q H_{\text {water }}\right)$.

To eliminate the source force density $f$, and thereby obtain a closed-form expression for the velocity power spectrum, we leverage the force-balance condition for the active nematic film alone, without external fluids:

$$
\eta_{n} \nabla^{2} \boldsymbol{v}_{i}-\nabla P_{i}+\boldsymbol{f}=\mathbf{0} .
$$

Here, the subscript $i$ indicates that the active nematic film is isolated. As in Eq. (B1), $\boldsymbol{f}$ accounts for source force density due to elastic, flow-alignment, and active stresses, albeit now in the isolated film. The source force of the isolated film [in Eq. (B18)] coincides with that of the full problem with external fluid layers [in Eq. (B1)] only if we ignore flow-alignment effects. In this limit, $\boldsymbol{f}$ does not depend explicitly on the flow field, and hence it is insensitive to the presence of external fluid layers. To exploit this fact and therefore be able to derive a closed form for the velocity power spectrum, we first ignore the flow-alignment coupling.

Following Ref. [37], we take the curl of Eq. (B18) to obtain a Poisson equation for the vorticity field $\omega=\hat{z} \cdot(\boldsymbol{\nabla} \times \boldsymbol{v})$ :

$$
\nabla^{2} \omega_{i}=s(\boldsymbol{r}, t), \quad s(\boldsymbol{r}, t)=-\frac{1}{\eta_{n}} \hat{z} \cdot(\boldsymbol{\nabla} \times \boldsymbol{f}),
$$

where $s$ is the vorticity source due to nematic forces. In Fourier space, this equation is written as

$$
-q^{2} \tilde{\omega}_{i}(\boldsymbol{q})=\tilde{s}(\boldsymbol{q})=\frac{i}{\eta_{n}}\left(q_{y} \tilde{f}_{x}-q_{x} \tilde{f}_{y}\right) .
$$

Hence, the vorticity spectrum of the isolated active film is given by

$\left\langle\left|\tilde{\omega}_{i}(\boldsymbol{q})\right|^{2}\right\rangle=\frac{1}{\eta_{n}^{2} q^{4}}\left\langle q_{y}^{2}\left|\tilde{f}_{x}\right|^{2}+q_{x}^{2}\left|\tilde{f}_{y}\right|^{2}-q_{x} q_{y}\left(\tilde{f}_{x} \tilde{f}_{y}^{*}+\tilde{f}_{y} \tilde{f}_{x}^{*}\right)\right\rangle$.

Comparing to Eq. (B16), we obtain 


$$
\left\langle|\tilde{\boldsymbol{v}}(\boldsymbol{q})|^{2}\right\rangle=\frac{\eta_{n}^{2} q^{2}}{\Lambda^{2}(q)}\left\langle\left|\tilde{\omega}_{i}(\boldsymbol{q})\right|^{2}\right\rangle,
$$

with $\Lambda(q)$ given by Eq. (B17). As explained above, this result is exact in the limit of vanishing flow-alignment coupling. In the presence of flow alignment, Eq. (B22) defines a closed approximation whereby the nematic forces, both passive and active, are included exactly, while the flow-alignment forces are approximated by the hydrodynamics of the isolated problem.

Equation (B22) relates the velocity spectrum of the active nematic film coupled to the external fluid layers with the vorticity spectrum of an isolated active nematic film. For the latter, we can now make use of the mean-field theory introduced by Giomi [36], which is based on decomposing the vorticity field into a superposition of $N$ uncorrelated vortices. Based on simulation results, the theory assumes that each vortex has a vorticity $\omega_{v}$ independent of its size, and that vortex areas follow an exponential distribution with mean $a_{*}=\pi R_{*}^{2}$, where $R_{*}$ is the mean vortex radius. With these assumptions, Giomi's mean-field theory predicts [36]

$$
\left\langle\left|\tilde{\omega}_{i}(\boldsymbol{q})\right|^{2}\right\rangle=\frac{N \omega_{v}^{2} R_{*}^{4}}{8 \pi^{2}} e^{-q^{2} R_{*}^{2} / 2}\left[I_{0}\left(\frac{q^{2} R_{*}^{2}}{2}\right)-I_{1}\left(\frac{q^{2} R_{*}^{2}}{2}\right)\right],
$$

where $I_{0}$ and $I_{1}$ are modified Bessel functions of the first kind. Introducing this result into Eq. (B22), and into Eq. (B15), we obtain the kinetic energy spectrum,

$E(q)=\frac{B q R_{*}^{4} e^{-q^{2} R_{*}^{2} / 2}\left[I_{0}\left(q^{2} R_{*}^{2} / 2\right)-I_{1}\left(q^{2} R_{*}^{2} / 2\right)\right]}{\left[q+\eta_{\mathrm{oil}} / \eta_{n} \tanh \left(q H_{\mathrm{oil}}\right)+\eta_{\text {water }} / \eta_{n} \operatorname{coth}\left(q H_{\text {water }}\right)\right]^{2}}$,

where $B=N \omega_{v}^{2} /\left(32 \pi^{3} \mathcal{A}\right)$ is a prefactor related to the total enstrophy, and independent of both the wave number $q$ and the mean vortex radius $R_{*}$.

\section{Scaling laws}

Here, we extend the discussion of the predicted scaling regimes given in the main text. As in the main text, we consider a situation with just one external fluid layer and classify the scaling regimes in terms of three characteristic lengths: the mean vortex radius $R_{*}$, the viscous length $\ell_{v}=\eta_{n} / \eta_{\text {ext }}$, and the external layer thickness $H$. In the main text, we discuss the scaling regimes at scales much smaller than the layer thickness, $q H \gg 1$, summarizing our results in Fig. 2. Here, we give the results in the opposite limit, looking at scales much larger than the layer thickness, $q H \ll 1$. In this thin-layer limit, the scaling laws depend on the boundary condition of the external fluid at the nonactive interface. We considered both a no-slip boundary

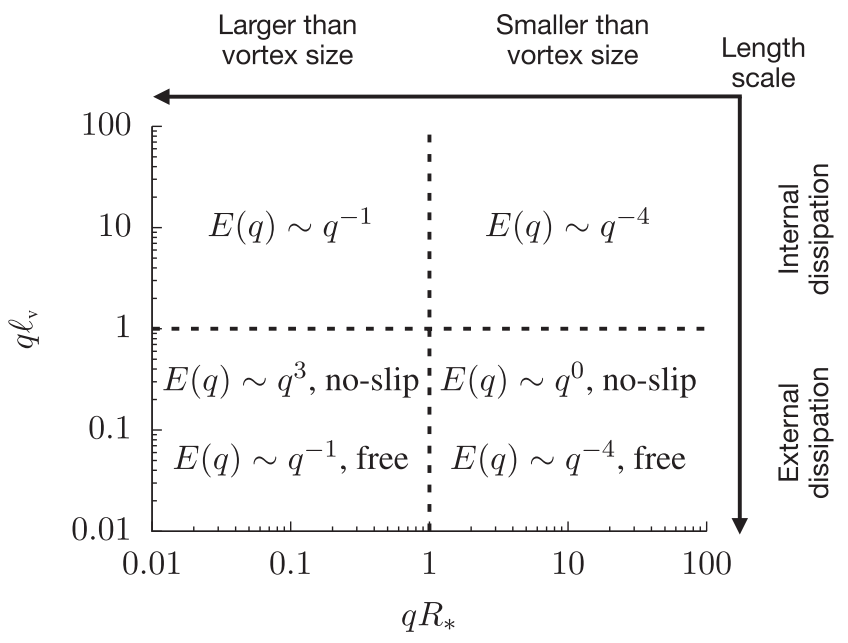

FIG. 6. Scaling regimes of turbulent flows in an active nematic film in contact with an external fluid. The different regimes are predicted at length scales $(\sim 1 / q)$ either larger or smaller than the mean vortex radius $R_{*}$, the viscous length $\ell_{v}=\eta_{n} / \eta_{\text {ext }}$, and the thickness $H$ of the external fluid layer. This figure summarizes the scalings in the thin-layer limit $q H \ll 1$; see the main text and Fig. 2 for the predictions in the thick-layer limit $q H \gg 1$.

condition, as for the water-substrate interface in our experiments, and a free surface, as for the oil-air interface in our experiments (Fig. 5). We summarize the results for all these situations in Fig. 6, which shows two additional scaling regimes, $E(q) \sim q^{3}$ and $E(q) \sim q^{0}$, for no-slip boundary conditions.

\section{APPENDIX C: VORTEX SIZE SELECTION}

Here, we discuss the physical origin of the mean vortex size $R_{*}$, a key parameter in the description of active turbulent flows. Active nematic turbulence results from the well-known spontaneous-flow instability in active nematic fluids $[1,2,4]$. Here, we ask whether vortex size is selected at the early stages of the instability, i.e., by its linear dynamics, or later on by the nonlinear dynamics.

In isolated active nematic films, the spontaneous-flow instability is a long-wavelength instability, whose growth rate is maximal at the longest wavelengths. As a result, the linear regime of the instability does not select any intrinsic wavelength but rather produces flow patterns with wavelengths determined by the system size. Indeed, system-sizedependent, stationary vortex patterns were observed in simulations at small activities, just past the instability threshold [37]. At high activity, however, these stationary vortices were unstable and evolved into turbulent flows. Simulations without flow alignment revealed a sequence of instabilities whereby vortices break into smaller vortices, down to a characteristic vortex size proportional to the critical wavelength of the instability $\lambda_{c}$, which is itself proportional to the active length $\ell_{a}$ [37]. In the presence of flow alignment, a similar transient cascade may exist. 
Moreover, flow alignment introduces a new nonlinearity in the problem enabling an additional potential mechanism of energy transfer in the steady state. It is thus reasonable to expect that, also in the presence of flow alignment, the vortex size for an isolated nematic is selected by a nonlinear mechanism.

This scenario is modified when the active nematic is not isolated but coupled either to a frictional substrate $[62,70]$ or to external fluids [24,53-55], as in our system. The external fluids are isotropic and in the Stokes regime, and therefore they cannot transfer energy across scales at the steady state. However, the coupling to an external fluid modifies the spontaneous-flow instability of the active nematic at the linear level, making the growth rate achieve a maximum at finite wavelengths [54,55]. Consequently, an intrinsic wavelength $\lambda_{m}$ is selected from the very onset of spontaneous flows, which dominates the early stages of turbulence development [24]. To what extent nonlinear effects may modify this characteristic scale at later stages of turbulence is an open question that we can now analyze in light of our data.

To this end, we derive the wavelength $\lambda_{m}$ that is selected in the linear regime by the coupling between the active nematic and the external fluids in our experiments. We then compare these predictions to our experimental measurements of the vortex size in fully developed turbulence at different oil viscosities.

To obtain the selected wavelength, we analyze the dynamics around the uniformly oriented quiescent state of the active nematic film. Ignoring flow alignment and topological defects, the dynamics of the angle $\theta$ of the nematic director field $\hat{\boldsymbol{n}}=(\cos \theta, \sin \theta)$ can be written as [37]

$$
\partial_{t} \theta+\boldsymbol{v} \cdot \boldsymbol{\nabla} \theta+\frac{\omega}{2}=\frac{K}{\gamma} \nabla^{2} \theta
$$

The right-hand side of this equation accounts for the relaxation of distortions of the director field, which generate elastic nematic stress. We describe this stress in the approximation of one Frank constant $K$ which, together with the rotational viscosity $\gamma$, controls the director relaxation rate [71]. Respectively, the second and third terms on the lefthand side of Eq. (C1) account for the advection and corotation of the nematic director by the flow field $v$, with vorticity $\omega=\hat{z} \cdot(\nabla \times v)$.

In turn, the flow field is driven by nematic forces $f$ as specified by the force balance Eq. (B1). Ignoring flow alignment as in Eq. (C1), the nematic forces are given by

$$
f_{\alpha}=\partial_{\beta}\left(\sigma_{\alpha \beta}^{\mathrm{ant}}+\sigma_{\alpha \beta}^{\mathrm{act}}\right)
$$

The first contribution accounts for elastic nematic stresses described by the antisymmetric part of the stress tensor [71],

$$
\sigma_{\alpha \beta}^{\mathrm{ant}}=\frac{1}{2}\left(n_{\alpha} h_{\beta}-n_{\beta} h_{\alpha}\right) .
$$

Here, $\boldsymbol{h}=-\delta F_{n} / \delta \hat{\boldsymbol{n}}=K \nabla^{2} \hat{\boldsymbol{n}}$ is the molecular field computed from the Frank free energy for nematic elasticity which, in the one-constant approximation, is given by [71]

$$
F_{n}=\frac{K}{2} \int\left(\partial_{\alpha} n_{\beta}\right)\left(\partial_{\alpha} n_{\beta}\right) d^{2} \boldsymbol{r}=\frac{K}{2} \int|\nabla \theta|^{2} d^{2} \boldsymbol{r} .
$$

Respectively, the second contribution in Eq. (C2) corresponds to the active nematic stress [4,72-74],

$$
\sigma_{\alpha \beta}^{\mathrm{act}}=-\zeta q_{\alpha \beta},
$$

where $\zeta$ is the active stress coefficient, and $q_{\alpha \beta}=n_{\alpha} n_{\beta}-$ $1 / 2 \delta_{\alpha \beta}$ is the nematic orientation tensor in two dimensions, with Cartesian components:

$$
\begin{gathered}
q_{x x}=-q_{y y}=\frac{1}{2} \cos (2 \theta), \\
q_{x y}=q_{y x}=\frac{1}{2} \sin (2 \theta) .
\end{gathered}
$$

To perform a linear stability analysis, we introduce perturbations around the reference state as $\theta=0+\delta \theta$ and $\boldsymbol{v}=\mathbf{0}+\delta \boldsymbol{v}$, and we decompose them into Fourier-Laplace modes as

$$
\begin{aligned}
& \delta \theta(\boldsymbol{r}, t)=\int \frac{d \Omega}{2 \pi} \int \frac{d^{2} \boldsymbol{q}}{(2 \pi)^{2}} \delta \tilde{\theta}(\boldsymbol{q}, \Omega) e^{\Omega(+i \boldsymbol{q} \cdot \boldsymbol{r}} \\
& \delta \boldsymbol{v}(\boldsymbol{r}, t)=\int \frac{d \Omega}{2 \pi} \int \frac{d^{2} \boldsymbol{q}}{(2 \pi)^{2}} \delta \tilde{\boldsymbol{v}}(\boldsymbol{q}, \Omega) e^{\Omega t+i \boldsymbol{q} \cdot \boldsymbol{r}}
\end{aligned}
$$

with wave vector $\boldsymbol{q}$ and frequency $\Omega$. In terms of these modes, and to first order in perturbations, Eq. (C1) is written as

$$
\Omega \delta \tilde{\theta}+\frac{1}{2}\left(i q_{y} \delta \tilde{v}_{x}-i q_{x} \delta \tilde{v}_{x}\right)=-\frac{K}{\gamma} q^{2} \delta \tilde{\theta} .
$$

In turn, the Fourier modes of the flow field are related to those of the nematic force $\tilde{f}$ via Eqs. (B3) and (B11). To first order in perturbations, Eqs. (C2)-(C6) yield

$$
\begin{aligned}
& \delta \tilde{f}_{x}=i q_{y}\left(-\zeta-\frac{K}{2} q^{2}\right) \delta \tilde{\theta} \\
& \delta \tilde{f}_{y}=i q_{x}\left(-\zeta+\frac{K}{2} q^{2}\right) \delta \tilde{\theta}
\end{aligned}
$$

Introducing these results into Eq. (B3), using Eq. (B11), and then introducing the resulting $\delta \tilde{v}$ into Eq. (C8), we obtain the growth rate of perturbations: 

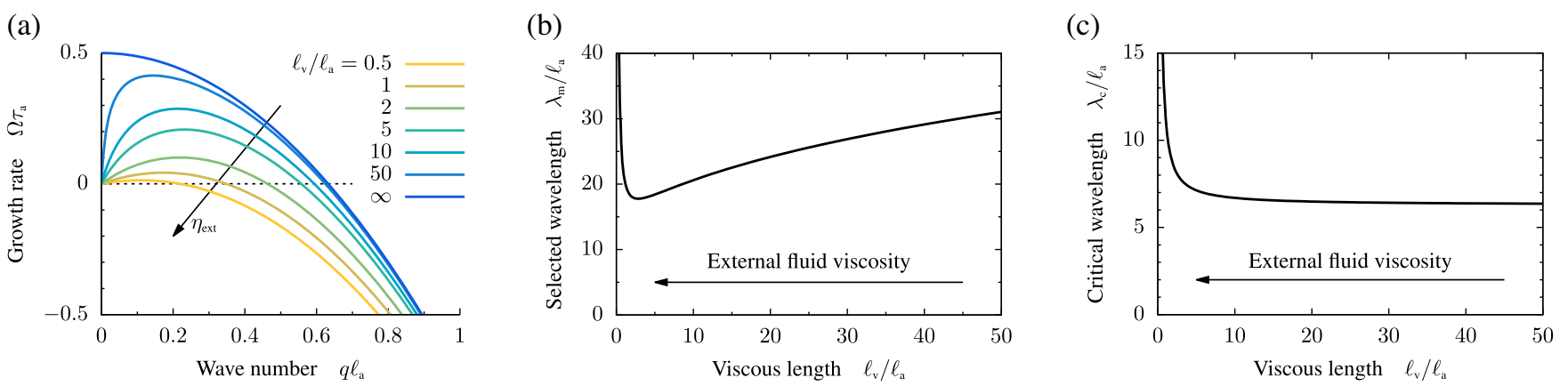

FIG. 7. Vortex size selection. (a) Growth rate [Eq. (C13)] along the most unstable direction for the spontaneous-flow instability in an active nematic film coupled to an external fluid layer. The viscosity ratio is set to $r=\gamma / \eta_{n}=1$, and flow alignment is ignored. The different curves correspond to different values of the external fluid viscosity $\eta_{\text {ext }}$, expressed in terms of the viscous length $\ell_{v}=\eta_{n} / \eta_{\text {ext }}$. The blue line corresponds to an isolated active nematic film, without external fluid. Lengths and time are rescaled by the active length and time, $\ell_{a}$ and $\tau_{a}$ defined in Eq. (C12). (b) Wavelength at which the growth rate is maximum, as obtained from Eq. (C14), as a function of the viscous length. This wavelength is selected by the linear dynamics upon the spontaneous-flow instability. (c) Critical wavelength of the spontaneous-flow instability [Eq. (C15)] as a function of the viscous length.

$$
\Omega(\boldsymbol{q})=-\frac{K}{\gamma} q^{2}+\frac{\zeta q^{2} \cos (2 \phi) / 2-K q^{4} / 4}{\Lambda(q)}
$$

where $\phi$ is the angle formed by the wave vector $\boldsymbol{q}$ and the director $\hat{\boldsymbol{n}}$, such that $\boldsymbol{q} \cdot \hat{\boldsymbol{n}}=q \cos \phi$, and $\Lambda(q)$ is the hydrodynamic kernel given by Eq. (B17).

To get insight into wavelength selection, we consider a situation with only one external fluid layer with thickness $H \rightarrow \infty$ and viscous length $\ell_{v}=\eta_{n} / \eta_{\mathrm{ext}}$. In this situation, Eq. (C10) takes the simpler form:

$$
\Omega(\boldsymbol{q})=-\frac{K}{\gamma} q^{2}+\frac{1}{\eta_{\mathrm{ext}}} \frac{\zeta q^{2} \cos (2 \phi) / 2-K q^{4} / 4}{\ell_{v} q^{2}+q} .
$$

Rescaling length and time by the active length and time, respectively,

$$
\ell_{a}=\sqrt{\frac{K}{|\zeta|} \frac{\eta_{n}}{\gamma}}, \quad \tau_{a}=\frac{\gamma}{K} \ell_{a}^{2}=\frac{\eta_{n}}{|\zeta|},
$$

the growth rate Eq. (C11) can be expressed in dimensionless form as

$\bar{\Omega}(q, \phi)=-\bar{q}^{2}+\frac{\operatorname{sgn}(\zeta) \bar{q}^{2} \cos (2 \phi) / 2-r \bar{q}^{4} / 4}{\bar{q}^{2}+\bar{q} / \bar{\ell}_{v}}$.

Here, $\bar{\Omega}=\Omega \tau_{a}$, and $\bar{q}=q \ell_{a}$ are dimensionless variables. We have also introduced three dimensionless parameters: the viscosity ratio $r \equiv \gamma / \eta_{n}$, the dimensionless viscous length $\bar{\ell}_{v}=\ell_{v} / \ell_{a}$, and the sign of the active stress $\operatorname{sgn}(\zeta)= \pm 1$ for extensile and contractile stresses, respectively.

The direction of most unstable perturbations is along the director $\left(\phi^{*}=0\right)$ for extensile stresses $(\zeta>0)$, and perpendicular to the director $\left(\phi^{*}=\pi / 2\right)$ for contractile stresses $(\zeta<0)$. Along the most unstable direction, the growth rate Eq. (C13) has the shape shown in Fig. 7(a). This figure shows that the coupling of the active nematic to the external fluid layer, represented by a finite $\bar{\ell}_{v}=\ell_{v} / \ell_{a}$, produces a maximum of the growth rate at a finite wavelength. This selected wavelength, $\lambda_{m}=2 \pi / q_{m}$, is obtained from the single real solution of the following cubic equation:

$$
\left(2+\frac{r}{2}\right) \bar{\ell}_{v}^{2} \bar{q}_{m}^{3}+\left(4+\frac{3 r}{4}\right) \bar{\ell}_{v} \bar{q}_{m}^{2}+2 \bar{q}_{m}-\frac{1}{2} \bar{\ell}_{v}=0 .
$$

As shown in Fig. 7(b), and also apparent in Fig. 7(a), the selected wavelength $\lambda_{m}$ has a nonmonotonic dependence on the viscous length $\ell_{v}$. In the common situation in which the viscous length is larger than the active length, the selected wavelength decreases with the viscosity of the external fluid. However, when the viscous length becomes smaller than the active length, the selected wavelength increases with the viscosity of the external fluid.

The coupling to the external fluid not only gives rise to the selected wavelength $\lambda_{m}$ but also modifies the critical wavelength of the instability, $\lambda_{c}=2 \pi / q_{c}$. This wavelength is determined by the condition $\Omega\left(q_{c}\right)=0$ along the most unstable direction, which gives

$q_{c}=\frac{1}{(2+r / 2) \ell_{v}}\left[-1+\sqrt{1+(2+r / 2) \frac{\ell_{v}^{2}}{\ell_{a}^{2}}}\right]$,

with $\ell_{a}$ given in Eq. (C12). As shown in Fig. 7(c), the critical wavelength $\lambda_{c}$ increases monotonically with the viscosity $\eta_{\text {ext }}$ of the external fluid. At small $\eta_{\text {ext }}$, i.e., large $\ell_{v}=\eta_{n} / \eta_{\text {ext }}$, the critical wavelength saturates at its value for isolated nematic films: 


$$
\lim _{\ell_{v} \gg \ell_{a}} \lambda_{c}=2 \pi \sqrt{2+r / 2} \ell_{a} .
$$

In the opposite limit, when dissipation is dominated by the viscosity of the external fluid, the critical wavelength becomes proportional to the external fluid viscosity:

$$
\lim _{\ell_{v} \ll \ell_{a}} \lambda_{c}=4 \pi \ell_{a} \frac{1}{\bar{\ell}_{v}}=4 \pi \ell_{a}^{2} \frac{\eta_{\mathrm{ext}}}{\eta_{n}} .
$$

In summary, the coupling to the external fluid endows the spontaneous flow instability with a linear wavelengthselection mechanism. This mechanism selects the characteristic scale of turbulent flows at early stages [24]. However, nonlinear effects may modify the selected scale at later stages, and thereby dictate the vortex size in the stationary, fully developed turbulent regime. To assess this point in our experiments, we analyze how stationary vortex size varies with oil viscosity.

If stationary vortex size were dictated by the linear wavelength-selection mechanism, vortex size should vary with oil viscosity as in Fig. 7(b). In our experiments, the oil viscous length is $\ell_{\text {oil }} \gtrsim R_{*}$, and the vortex size should be $R_{*} \gtrsim \ell_{a}$. Therefore, we are in the regime $\ell_{v}>\ell_{a}$, and vortex size should moderately decrease with oil viscosity [Fig. 7(b)]. Instead, if vortex size were dictated by the nonlinear selection mechanism of Ref. [37], vortex size should be proportional to the critical wavelength, and therefore vary with oil viscosity as in Fig. 7(c). Thus, again in the regime $\ell_{v}>\ell_{a}$, vortex size should remain roughly independent of oil viscosity [Fig. 7(c)].

Our experimental measurements show that the mean vortex radius, as measured from the vortex area distribution, is rather independent of oil viscosity over a range spanning several orders of magnitude of oil viscosity [Fig. 4(b)]. At very high oil viscosities, we observe a moderate decrease of vortex radius [Fig. 4(b)], whose interpretation remains an open question.

This analysis suggests that the linear selection mechanism alone does not explain the observed vortex size in the stationary regime, thus implying some form of energy transfer across scales, be it transient and/or steady. Elucidating the mechanism of vortex size selection in more detail thus remits to fundamental open questions in active turbulence that we defer to future work.

[1] R. A. Simha and S. Ramaswamy, Hydrodynamic Fluctuations and Instabilities in Ordered Suspensions of SelfPropelled Particles, Phys. Rev. Lett. 89, 058101 (2002).

[2] R. Voituriez, J. F. Joanny, and J. Prost, Spontaneous Flow Transition in Active Polar Gels, Europhys. Lett. 70, 404 (2005).

[3] R. Golestanian, J. M. Yeomans, and N. Uchida, Hydrodynamic Synchronization at Low Reynolds Number, Soft Matter 7, 3074 (2011).
[4] M. C. Marchetti, J. F. Joanny, S. Ramaswamy, T. B. Liverpool, J. Prost, M. Rao, and R. A. Simha, Hydrodynamics of Soft Active Matter, Rev. Mod. Phys. 85, 1143 (2013).

[5] N. Uchida and R. Golestanian, Synchronization and Collective Dynamics in a Carpet of Microfluidic Rotors, Phys. Rev. Lett. 104, 178103 (2010).

[6] S. P. Thampi, R. Golestanian, and J. M. Yeomans, Vorticity, Defects and Correlations in Active Turbulence, Phil. Trans. R. Soc. A 372, 20130366 (2014).

[7] A. Doostmohammadi, J. Ignés-Mullol, J. M. Yeomans, and F. Sagués, Active Nematics, Nat. Commun. 9, 3246 (2018).

[8] R. Alert, J. Casademunt, and J.-F. Joanny, Active Turbulence, arXiv:2104.02122.

[9] C. Dombrowski, L. Cisneros, S. Chatkaew, R. E. Goldstein, and J.O. Kessler, Self-Concentration and Large-Scale Coherence in Bacterial Dynamics, Phys. Rev. Lett. 93, 098103 (2004).

[10] L. H. Cisneros, R. Cortez, C. Dombrowski, R. E. Goldstein, and J. O. Kessler, Fluid Dynamics of Self-Propelled Microorganisms, from Individuals to Concentrated Populations, Exp. Fluids 43, 737 (2007).

[11] T. Ishikawa, N. Yoshida, H. Ueno, M. Wiedeman, Y. Imai, and T. Yamaguchi, Energy Transport in a Concentrated Suspension of Bacteria, Phys. Rev. Lett. 107, 028102 (2011).

[12] H. H. Wensink, J. Dunkel, S. Heidenreich, K. Drescher, R. E. Goldstein, H. Löwen, and J. M. Yeomans, Meso-Scale Turbulence in Living Fluids, Proc. Natl. Acad. Sci. U.S.A. 109, 14308 (2012).

[13] J. Dunkel, S. Heidenreich, K. Drescher, H. H. Wensink, M. Bär, and R.E. Goldstein, Fluid Dynamics of Bacterial Turbulence, Phys. Rev. Lett. 110, 228102 (2013).

[14] A. E. Patteson, A. Gopinath, and P. E. Arratia, The Propagation of Active-Passive Interfaces in Bacterial Swarms, Nat. Commun. 9, 5373 (2018).

[15] H. Li, X.-Q. Shi, M. Huang, X. Chen, M. Xiao, C. Liu, H. Chaté, and H. P. Zhang, Data-Driven Quantitative Modeling of Bacterial Active Nematics, Proc. Natl. Acad. Sci. U.S.A. 116, 777 (2019).

[16] Y. Peng, Z. Liu, and X. Cheng, Imaging the Emergence of Bacterial Turbulence: Phase Diagram and Transition Kinetics, Sci. Adv. 7, eabd1240 (2021).

[17] Z. Liu, W. Zeng, X. Ma, and X. Cheng, Density Fluctuations and Energy Spectra of 3D Bacterial Suspensions, arXiv:2012.13680.

[18] A. Creppy, O. Praud, X. Druart, P. L. Kohnke, and F. Plouraboué, Turbulence of Swarming Sperm, Phys. Rev. E 92, 032722 (2015).

[19] T. Sanchez, D. T. N. Chen, S. J. DeCamp, M. Heymann, and Z. Dogic, Spontaneous Motion in Hierarchically Assembled Active Matter, Nature (London) 491, 431 (2012).

[20] G. Henkin, S. J. DeCamp, D. T. N. Chen, T. Sanchez, and Z. Dogic, Tunable Dynamics of Microtubule-Based Active Isotropic Gels, Phil. Trans. R. Soc. A 372, 20140142 (2014).

[21] P. Guillamat, J. Ignés-Mullol, and F. Sagués, Taming Active Turbulence with Patterned Soft Interfaces, Nat. Commun. 8, 564 (2017). 
[22] P. W. Ellis, D. J. G. Pearce, Y.-W. Chang, G. Goldsztein, L. Giomi, and A. Fernandez-Nieves, Curvature-Induced Defect Unbinding and Dynamics in Active Nematic Toroids, Nat. Phys. 14, 85 (2018).

[23] L. M. Lemma, S. J. DeCamp, Z. You, L. Giomi, and Z. Dogic, Statistical Properties of Autonomous Flows in 2D Active Nematics, Soft Matter 15, 3264 (2019).

[24] B. Martínez-Prat, J. Ignés-Mullol, J. Casademunt, and F. Sagués, Selection Mechanism at the Onset of Active Turbulence, Nat. Phys. 15, 362 (2019).

[25] A. J. Tan, E. Roberts, S. A. Smith, U. A. Olvera, J. Arteaga, S. Fortini, K. A. Mitchell, and L.S. Hirst, Topological Chaos in Active Nematics, Nat. Phys. 15, 1033 (2019).

[26] G. Duclos, R. Adkins, D. Banerjee, M. S. E. Peterson, M. Varghese, I. Kolvin, A. Baskaran, R. A. Pelcovits, T. R. Powers, A. Baskaran, F. Toschi, M. F. Hagan, S. J. Streichan, V. Vitelli, D. A. Beller, and Z. Dogic, Topological Structure and Dynamics of Three-Dimensional Active Nematics, Science 367, 1120 (2020).

[27] A. Doostmohammadi, S. P. Thampi, T. B. Saw, C. T. Lim, B. Ladoux, and J. M. Yeomans, Cell Division: A Source of Active Stress in Cellular Monolayers, Soft Matter 11, 7328 (2015).

[28] T. D. Yang, H. Kim, C. Yoon, S.-K. Baek, and K. J. Lee, Collective Pulsatile Expansion and Swirls in Proliferating Tumor Tissue, New J. Phys. 18, 103032 (2016).

[29] C. Blanch-Mercader, V. Yashunsky, S. Garcia, G. Duclos, L. Giomi, and P. Silberzan, Turbulent Dynamics of Epithelial Cell Cultures, Phys. Rev. Lett. 120, 208101 (2018).

[30] D. Nishiguchi and M. Sano, Mesoscopic Turbulence and Local Order in Janus Particles Self-Propelling under an ac Electric Field, Phys. Rev. E 92, 052309 (2015).

[31] G. Kokot, S. Das, R. G. Winkler, G. Gompper, I. S. Aranson, and A. Snezhko, Active Turbulence in a Gas of Self-Assembled Spinners, Proc. Natl. Acad. Sci. U.S.A. 114, 12870 (2017).

[32] H. Karani, G. E. Pradillo, and P. M. Vlahovska, Tuning the Random Walk of Active Colloids: From Individual Runand-Tumble to Dynamic Clustering, Phys. Rev. Lett. 123, 208002 (2019).

[33] M. Bourgoin, R. Kervil, C. Cottin-Bizonne, F. Raynal, R. Volk, and C. Ybert, Kolmogorovian Active Turbulence of a Sparse Assembly of Interacting Marangoni Surfers, Phys. Rev. X 10, 021065 (2020).

[34] A. N. Kolmogorov, The Local Structure of Turbulence in Incompressible Viscous Fluid for Very Large Reynolds Numbers, Proc. R. Soc. A 434, 9 (1991).

[35] U. Frisch, Turbulence. The Legacy of A.N. Kolmogorov (Cambridge University Press, Cambridge, England, 1995).

[36] L. Giomi, Geometry and Topology of Turbulence in Active Nematics, Phys. Rev. X 5, 031003 (2015).

[37] R. Alert, J.-F. Joanny, and J. Casademunt, Universal Scaling of Active Nematic Turbulence, Nat. Phys. 16, 682 (2020).

[38] V. Bratanov, F. Jenko, and E. Frey, New Class of Turbulence in Active Fluids, Proc. Natl. Acad. Sci. U.S.A. 112, 15048 (2015).

[39] M. Linkmann, G. Boffetta, M. C. Marchetti, and B. Eckhardt, Phase Transition to Large Scale Coherent Structures in Two-Dimensional Active Matter Turbulence, Phys. Rev. Lett. 122, 214503 (2019).
[40] D. Bárdfalvy, H. Nordanger, C. Nardini, A. Morozov, and J. Stenhammar, Particle-Resolved Lattice Boltzmann Simulations of 3-Dimensional Active Turbulence, Soft Matter 15, 7747 (2019).

[41] S. P. Thampi, R. Golestanian, and J. M. Yeomans, Velocity Correlations in an Active Nematic, Phys. Rev. Lett. 111, 118101 (2013).

[42] J. Urzay, A. Doostmohammadi, and J. M. Yeomans, MultiScale Statistics of Turbulence Motorized by Active Matter, J. Fluid Mech. 822, 762 (2017).

[43] L. N. Carenza, L. Biferale, and G. Gonnella, Multiscale Control of Active Emulsion Dynamics, Phys. Rev. Fluids 5, 011302(R) (2020).

[44] R. C. V. Coelho, N. A. M. Araújo, and M. M. T. da Gama, Propagation of Active Nematic-Isotropic Interfaces on Substrates, Soft Matter 16, 4256 (2020).

[45] Ž. Krajnik, Ž. Kos, and M. Ravnik, Spectral Energy Analysis of Bulk Three-Dimensional Active Nematic Turbulence, Soft Matter 16, 9059 (2020).

[46] R. Großmann, P. Romanczuk, M. Bär, and L. SchimanskyGeier, Vortex Arrays and Mesoscale Turbulence of SelfPropelled Particles, Phys. Rev. Lett. 113, 258104 (2014).

[47] J. Słomka and J. Dunkel, Spontaneous Mirror-Symmetry Breaking Induces Inverse Energy Cascade in 3D Active Fluids, Proc. Natl. Acad. Sci. U.S.A. 114, 2119 (2017).

[48] M. James, W. J. T. Bos, and M. Wilczek, Turbulence and Turbulent Pattern Formation in a Minimal Model for Active Fluids, Phys. Rev. Fluids 3, 061101(R) (2018).

[49] Sanjay C. P. and A. Joy, Friction Scaling Laws for Transport in Active Turbulence, Phys. Rev. Fluids 5, 024302 (2020).

[50] G. Boffetta, A. Cenedese, S. Espa, and S. Musacchio, Effects of Friction on 2D Turbulence: An Experimental Study of the Direct Cascade, Europhys. Lett. 71, 590 (2005).

[51] G. Boffetta and R. E. Ecke, Two-Dimensional Turbulence, Annu. Rev. Fluid Mech. 44, 427 (2012).

[52] See Supplemental Material at http://link.aps.org/ supplemental/10.1103/PhysRevX.11.031065 for additional supporting figures and for supporting videos of the experiments.

[53] N. Sarkar and A. Basu, Role of Interfacial Friction for Flow Instabilities in a Thin Polar-Ordered Active Fluid Layer, Phys. Rev. E 92, 052306 (2015).

[54] T. Gao, R. Blackwell, M. A. Glaser, M. D. Betterton, and M. J. Shelley, Multiscale Polar Theory of Microtubule and Motor-Protein Assemblies, Phys. Rev. Lett. 114, 048101 (2015).

[55] T. Gao, M. D. Betterton, A.-S. Jhang, and M. J. Shelley, Analytical Structure, Dynamics, and Coarse Graining of a Kinetic Model of an Active Fluid, Phys. Rev. Fluids 2, 093302 (2017).

[56] P. Guillamat, J. Ignés-Mullol, S. Shankar, M. C. Marchetti, and F. Sagués, Probing the Shear Viscosity of an Active Nematic Film, Phys. Rev. E 94, 060602(R) (2016).

[57] R. Matas-Navarro, R. Golestanian, T. B. Liverpool, and S. M. Fielding, Hydrodynamic Suppression of Phase Separation in Active Suspensions, Phys. Rev. E 90, 032304 (2014).

[58] H. A. Stone and H. McConnell, Hydrodynamics of Quantized Shape Transitions of Lipid Domains, Proc. R. Soc. A 448, 97 (1995). 
[59] H. A. Stone and H. M. McConnell, Lipid Domain Instabilities in Monolayers Overlying Sublayers of Finite Depth, J. Phys. Chem. 99, 13505 (1995).

[60] D. K. Lubensky and R. E. Goldstein, Hydrodynamics of Monolayer Domains at the Air-Water Interface, Phys. Fluids 8, 843 (1996).

[61] D. P. Rivas, T. N. Shendruk, R. R. Henry, D. H. Reich, and R. L. Leheny, Driven Topological Transitions in Active Nematic Films, Soft Matter 16, 9331 (2020).

[62] S. P. Thampi, R. Golestanian, and J. M. Yeomans, Active Nematic Materials with Substrate Friction, Phys. Rev. E 90, 062307 (2014).

[63] E. J. Hemingway, M. E. Cates, and S. M. Fielding, Viscoelastic and Elastomeric Active Matter: Linear Instability and Nonlinear dynamics, Phys. Rev. E 93, 032702 (2016).

[64] L. Grunberg and A. H. Nissan, Mixture Law for Viscosity, Nature (London) 164, 799 (1949).

[65] B. Zhmud and M. Roegiers, Property Blending Relationships for Binary Mixtures of Mineral Oil and Elektrionised Vegetable Oil: Viscosity, Solvent Power, and Seal Compatibility Index, Lubr. Sci. 23, 263 (2011).

[66] A. D. Edelstein, M. A. Tsuchida, N. Amodaj, H. Pinkard, R. D. Vale, and N. Stuurman, Advanced Methods of Microscope Control Using $\mu$ Manager Software, J. Biol. Methods 1, e10 (2014).

[67] J. Schindelin, I. Arganda-Carreras, E. Frise, V. Kaynig, M. Longair, T. Pietzsch, S. Preibisch, C. Rueden, S. Saalfeld, B. Schmid, J. Y. Tinevez, D. J. White, V. Hartenstein, K. Eliceiri, P. Tomancak, and A. Cardona, Fiji: An
Open-Source Platform for Biological-Image Analysis, Nat. Methods 9, 676 (2012).

[68] W. Thielicke and E. J. Stamhuis, PIVlab-Towards UserFriendly, Affordable and Accurate Digital Particle Image Velocimetry in MATLAB, J. Open Res. Software 2, e30 (2014).

[69] D. Garcia, Robust Smoothing of Gridded Data in One and Higher Dimensions with Missing Values, Computational Statistics and Data Analysis 54, 1167 (2010).

[70] G. Duclos, C. Blanch-Mercader, V. Yashunsky, G. Salbreux, J.-F. Joanny, J. Prost, and P. Silberzan, Spontaneous Shear Flow in Confined Cellular Nematics, Nat. Phys. 14, 728 (2018).

[71] P.-G. de Gennes and J. Prost, The Physics of Liquid Crystals, 2nd ed. (Oxford University Press, New York, 1993).

[72] K. Kruse, J. F. Joanny, F. Jülicher, J. Prost, and K. Sekimoto, Generic Theory of Active Polar Gels: A Paradigm for Cytoskeletal Dynamics, Eur. Phys. J. E 16, 5 (2005).

[73] J. Prost, F. Jülicher, and J.-F. Joanny, Active Gel Physics, Nat. Phys. 11, 111 (2015).

[74] F. Jülicher, S. W. Grill, and G. Salbreux, Hydrodynamic Theory of Active Matter, Rep. Prog. Phys. 81, 076601 (2018).

Correction: The caption to Fig. 3 contained copyediting and proof change insertion errors and has been set right.

Second Correction: A grant number in the Acknowledgments section contained an error and has been fixed. 